\title{
Spontaneous CP violation and the strong CP problem
}

\section{Luca Vecchi} \\ Maryland Center for Fundamental Physics, Department of Physics, University of Maryland, \\ College Park, MD 20742, U.S.A. \\ E-mail: vecchi@umd.edu
}

ABSTRACT: We derive sufficient conditions that guarantee a robust solution of the strong $\mathrm{CP}$ problem in theories with spontaneous $\mathrm{CP}$ violation, and introduce a class of models satisfying these requirements. In the simplest scenarios the dominant contribution to the topological angle arises at 3-loop order in the Standard Model Yukawa couplings. A variety of new realizations are obtained on a warped extra dimension, which can dynamically generate a CKM phase of the right size as well as the Planck-TeV hierarchy. Experimental signatures of this approach to the strong $\mathrm{CP}$ problem include a characteristic pattern of flavor violation and vector-like partners of the top and/or bottom quarks.

Keywords: Beyond Standard Model, CP violation

ARXIV EPRINT: 1412.3805 


\section{Contents}

1 Motivation 1

2 Model-independent analysis $\quad 3$

2.1 The CKM phase 3

2.2 A small $\bar{\theta}$ ? 3

$\begin{array}{lll}2.2 .1 & \text { Generalization } & 7\end{array}$

3 A realistic model on $\mathrm{AdS}_{5} \quad 8$

3.1 Setup 8

$\begin{array}{lr}3.2 & \text { Minimal flavor and CP violation }\end{array}$

$\begin{array}{llr}3.3 & \theta_{\mathrm{CKM}} \sim 1 \text {, dynamically } & 10\end{array}$

3.4 CFT interpretation and estimate of $\bar{\theta} \quad 12$

4 Phenomenology 13

$\begin{array}{lll}4.1 & \text { Electroweak observables } & 13\end{array}$

$\begin{array}{lll}4.2 & \text { Flavor violation } & 14\end{array}$

$\begin{array}{lll}4.3 & \text { Neutron EDM from dimension-6 operators } & 16\end{array}$

$\begin{array}{ll}4.4 \text { Collider searches } & 16\end{array}$

$\begin{array}{lll}5 & \text { Discussion } & 17\end{array}$

$\begin{array}{ll}\text { A 4D models } & 19\end{array}$

$\begin{array}{ll}\text { B On the previous literature } & 20\end{array}$

$\begin{array}{ll}\text { C A minimal confining CP-violating sector } & 21\end{array}$

\section{Motivation}

The colored sector of the standard model (SM) has two CP-odd parameters of phenomenological interest, parametrized by two nearly RG-invariant phases. One is the CKM phase $\theta_{\mathrm{CKM}}$, defined in terms of the Jarlskog invariant. The second is the topological angle $\bar{\theta}=\theta_{\mathrm{QCD}}-\theta_{\mathrm{F}}$, with $\theta_{\mathrm{QCD}}$ the coefficient of $\frac{g_{s}^{2}}{32 \pi^{2}} G \widetilde{G}$ and $\theta_{\mathrm{F}}=\operatorname{Arg} \operatorname{det}\left(Y_{u} Y_{d}\right)$ a function of the SM Yukawa couplings $Y_{u} \bar{q} \widetilde{H} u+Y_{d} \bar{q} H d$. Experimentally we find that [1]

$$
|\bar{\theta}|<10^{-10} \quad \theta_{\mathrm{CKM}} \sim 1 .
$$

The first constraint follows from the current $90 \%$ CL bound on the neutron EDM, $\left|d_{n}\right| \leq$ $2.9 \times 10^{-26} e \mathrm{~cm}$ (note that the contribution of $\theta_{\mathrm{CKM}}$ to $d_{n}$ is negligible [2-4]). Unfortunately the relation between $d_{n}, \bar{\theta}$ is not known to better than an order of magnitude (see e.g. $[5,6]$ 
and references therein), so the constraint on $\bar{\theta}$ quoted in (1.1) - obtained using naive dimensional analysis (NDA) - should be interpreted at the order of magnitude level.

The experimental inputs (1.1) are at the heart of the so called strong CP problem. Such "problem" arises because there is overwhelming evidence that the Standard Model is incomplete, so whatever UV dynamics completes it must be non-generic in order to explain (1.1): how come $\bar{\theta}$ is so small despite the fact that $\theta_{\mathrm{CKM}} \sim 1$ implies that $\mathrm{CP}$ is not a symmetry of the ultimate theory?

UV models that attempt to solve the strong CP problem via new symmetry principles can be broadly classified into two categories:

- scenarios with a global U(1) with a color anomaly [7-10]. If the symmetry is spontaneously broken at some high scale and explicitly broken dominantly by nonperturbative QCD effects, then the $\bar{\theta}$ angle dynamically relaxes to zero, and the theory predicts a QCD axion. The $\bar{\theta}$ angle becomes unphysical if $\mathrm{U}(1)$ is not spontaneously broken. $^{1}$

- models with spontaneous CP and/or P violation [15-31] (see [32] for a review of the earlier literature). Here $\bar{\theta}=0$ in the UV, and gets generated after spontaneous breaking.

There are also examples in which both a mirror symmetry and an anomalous $\mathrm{U}(1)$ are present, e.g. [33, 34].

The attractive feature characterizing the QCD axion is that this approach does not restrict flavor nor CP violation in the UV. The problem is that in order to evade current laboratory and astrophysics bounds, the axion decay constant has been pushed in a very uncomfortable regime in which the axion potential becomes enormously sensitive to possible U(1)-breaking trans-Planckian effects [35]. To some extent, the QCD axion has lost part of its original appeal, and has started to look more like a remarkable accident of the physics at very short distances. It is therefore useful to investigate the plausibility of alternative solutions.

$\mathrm{CP}$ is a gauge symmetry in several extra-dimensional extensions of the SM, including critical string theories $[38,39]$. This means that one can build scenarios based on spontaneous CP violation in which quantum gravity poses no threat to the basic symmetry principle. The question however is whether and how CP is broken after compactification down to our 4 space-time dimensions. It is conceivable that there exists a large number of realistic vacua in which CP breaking can be modeled via an effective 4D Lagrangian, but no definite conclusion can be drawn without a concrete model and a theory of moduli stabilization.

Nevertheless, the important point is that from an effective 4D theory perspective it is perfectly sensible, and also very well motivated, to assume that CP is a good symmetry of the UV, spontaneously broken at a scale parametrically low compared to the Planck scale. In this paper we will explore the viability of these models from a low energy perspective.

\footnotetext{
${ }^{1}$ For earlier work on the controversial "missing up-quark mass solution" see [11]. This possibility is currently strongly disfavored by lattice data, see e.g. [14].
} 
We assume CP is spontaneously broken in a secluded color-neutral sector and communicated to the SM via messenger fields, and ask: what constraints should the messenger dynamics satisfy in order to simultaneously account for a small neutron EDM and a large CKM phase? In section 2 we present sufficient conditions for this to happen. Our modelindependent approach suggests novel solutions to the strong CP problem. In particular, warped extra dimensions offer a variety of ways to implement our requirements, as is shown with an explicit model in section 3. Section 4 highlights the main phenomenological signatures of spontaneous CP violation. A discussion of our results is given in section 5 .

\section{Model-independent analysis}

\subsection{The CKM phase}

Let us denote by $\Sigma$ the CP-odd scalars whose vacuum break CP spontaneously, and assume $\mathrm{CP}$ violation is communicated to the SM quarks via an interaction with mediators characterized by a mass scale $m_{*}$. Collectively denoting by $\lambda$ the SM-messenger couplings, the size of $\mathrm{CP}$ violation in the visible sector is expected to be controlled by the following complex parameter $\xi \equiv \frac{\lambda \Sigma}{m_{*}}$. Crucially, the second experimental evidence in (1.1) requires that some of these parameters satisfy

$$
\operatorname{Im}(\xi) \sim 1 .
$$

(More precisely, (2.1) should hold in the field basis in which all CP-even couplings are real. We will be more rigorous below.) This implies the existence of a large mixing between the SM quarks and the messenger dynamics.

We review in appendix A and B how (2.1) is realized in some of the existing literature. In the following we will assume that $\xi \sim 1$ has been arranged, and look at the first requirement in (1.1).

\section{$2.2 \quad$ A small $\bar{\theta}$ ?}

Finding reliable estimates for $\bar{\theta}$ is rather prohibitive, especially in view of two facts. First, the current bound is so stringent that even high-loop effects can spoil an otherwise brilliant solution. An accurate calculation would therefore require a study of multi-loop diagrams, which is technically challenging. Second, we just saw that $\theta_{\mathrm{CKM}} \sim 1$ implies a large quark-messenger mixing $\xi$ at the scale $m_{*}$, see (2.1), so no obvious expansion parameter is available at the matching scale between the SM and the UV physics.

Here we point out that one can take advantage of the selection rules associated to the SM flavor symmetry to identify robust sufficient conditions for a small $\bar{\theta}$ in a large class of non-Supersymmetric models (we will comment on SUSY in section 5). This formalism also naturally selects the SM Yukawa couplings as the relevant expansion parameter. By exploring different spurious charges of $\xi$ under the SM flavor symmetry we can in fact "estimate $\bar{\theta}$ " in a model-independent way and identify the features that realistic scenarios should possess. (It is important to emphasize that in this work all global symmetries are viewed as accidental and, in particular, the SM flavor symmetry is not assumed to be exact in the UV: as in the chiral Lagrangian, we merely use it as a tool to define our power counting.) 


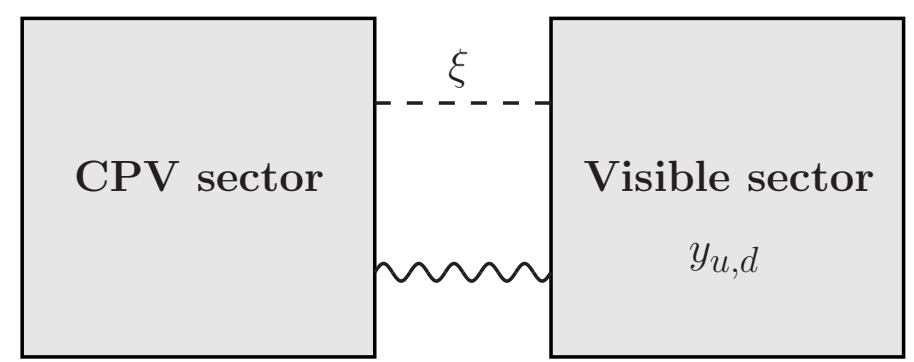

Figure 1. Schematic picture of the setup: CP is spontaneously broken in a color-neutral CPV sector and communicated to the visible sector via a CP-odd and flavor-violating vev $\xi \sim 1$. An example of minimal CPV sector is presented in appendix $\mathrm{C}$, while two models for the messenger dynamics are discussed in section 3 and appendix A (both belong to a more general class, see eq. (3.14)). As argued at the end of this section, flavor-violation beyond MFV is severely constrained by (1.1), whereas flavor-conserving interactions (wavy line) are not.

To illustrate our approach we start with a discussion of the most minimal class of models, in which the SM flavor symmetry is broken solely by

$$
y_{u}, \quad y_{d}, \quad \xi,
$$

with $y_{u, d}$ the SM (proto-)Yukawa couplings in the UV. The field basis we are working with is defined (up to orthogonal rotations of the SM fields) such that $\xi$ is a complex matrix, $\theta_{\mathrm{QCD}}=0$, whereas $y_{u, d}$ are real. This is the minimal set of flavor-violating parameters our models can have because $y_{u, d}$ are necessary to generate the SM quark masses without fine-tuning, ${ }^{2}$ and must be real in the absence of CP-violation. Our assumption (2.2) is equivalent to having 3 flavor-violating matrices: the true SM Yukawas $Y_{u, d}$ - obtained after having integrated out the messengers - and $\xi$. Our setup is summarized in figure 1. The generalization to less minimal scenarios goes in the direction of increasing $\bar{\theta}$, as argue at the end of this section.

We estimate $\bar{\theta}$ by matching the UV theory onto the SM at the messenger scale $m_{*} \gg$ $100 \mathrm{GeV}$. In this approach there are two types of contributions to $\bar{\theta}$. First, there are UVsensitive local contributions, that appear as polynomials of $y_{u, d}, \xi$ with real coefficients. ${ }^{3}$ Second, there are UV-finite corrections, which may contain more complicated functions (e.g. $\operatorname{logs}$ ) of $y_{u, d}, \xi$. We postulate that the exotic fields have masses $\geq m_{*}$ that remain finite as $y, \xi \rightarrow 0$. This assumption guarantees that all UV-finite effects decouple as $m_{*} \rightarrow \infty$ (and also that the CKM is unitary up to $m_{\mathrm{SM}}^{2} / m_{*}^{2}$ terms, see section 4 ). We will therefore analyze the more dangerous non-decoupling effects, that are polynomial contributions to $\bar{\theta}$ that survive even in the decoupling limit.

In our language $\bar{\theta}$ is a CP-odd and flavor-singlet function of (2.2). This must arise as a combination of $\mathrm{CP}$-even new physics couplings, CP-even Yukawas $y_{u, d}$, and the sole

\footnotetext{
${ }^{2}$ To avoid tuning, the quark masses must vanish with some parameter $Y$ that runs according to the RG equation $\beta_{Y} \propto Y+\mathcal{O}\left(Y^{3}\right)$. It will soon be clear that $Y$ cannot be a function of $\xi$ only, because in that case $\xi$ should be in the bi-fundamental of the SM flavor group and it would be virtually impossible to make $\bar{\theta}$ small. In this sense the choice (2.2) is the most minimal.

${ }^{3}$ In principle, non-perturbative real functions may also appear, but this does not affect our conclusions.
} 


\begin{tabular}{|c|c|c|}
\hline$\xi$ & Leading CP-odd structure & NDA estimate of $\bar{\theta}$ \\
\hline $\mathbf{3}, \mathbf{8} \in \mathrm{SU}(3)_{q}$ & $\operatorname{Im} \operatorname{tr}\left\{f_{1}\left(y_{u} y_{u}^{\dagger}\right) f_{2}\left(y_{d} y_{d}^{\dagger}\right)-(1 \leftrightarrow 2)\right\}$ & $\lambda_{C}^{2} \frac{Y_{t}^{2}}{4 \pi^{2}} \frac{Y_{b}^{2}}{4 \pi^{2}} \sim 5 \times 10^{-9}$ \\
\hline $\mathbf{3} \in \mathrm{SU}(3)_{u}$ & $\operatorname{Im}\left\{\xi^{\dagger}\left[y_{u}^{\dagger} y_{u}, y_{u}^{\dagger} y_{d} y_{d}^{\dagger} y_{u}\right] \xi\right\}$ & $\lambda_{C}^{2}\left(\frac{Y_{t}^{2}}{4 \pi^{2}}\right)^{2} \frac{Y_{b}^{2}}{4 \pi^{2}} \frac{Y_{c}}{Y_{t}} \sim 3 \times 10^{-13}$ \\
$\left(\frac{Y_{t}^{2}}{4 \pi^{2}}\right)^{2} \frac{Y_{c}^{2}}{4 \pi^{2}} \sim 8 \times 10^{-11}$ \\
$\mathbf{8} \in \mathrm{SU}(3)_{u}$ & $\operatorname{Im} \operatorname{tr}\left\{\left(y_{u}^{\dagger} y_{u}\right)^{2} \xi\left[y_{u}^{\dagger} y_{u}, \xi\right] \xi\right\}$ & $\lambda_{C}^{2} \frac{Y_{t}^{2}}{4 \pi^{2}}\left(\frac{Y_{b}^{2}}{4 \pi^{2}}\right)^{2} \frac{Y_{s}}{Y_{b}} \sim 5 \times 10^{-16}$ \\
\hline $\mathbf{3 , 8} \in \mathrm{SU}(3)_{d}$ & $\operatorname{Im} \operatorname{tr}\left\{f_{1}\left(y_{d}^{\dagger} y_{u} y_{u}^{\dagger} y_{d}\right) f_{2}\left(y_{d}^{\dagger} y_{d}\right)-(1 \leftrightarrow 2)\right\}$ &
\end{tabular}

Table 1. Leading non-decoupling contributions to $\bar{\theta}$ in an expansion in $O\left(Y^{2}\right)$ for the models of figure 1 , with various representations of the $\mathrm{CP}$-odd spurion $\xi$. The $f$ s are real functions of $\xi$, with $f_{1} \neq f_{2}$. In the last column we estimated $\bar{\theta}$ using NDA, with the SM Yukawas $Y_{u, d}$ renormalized at $1 \mathrm{TeV}, \lambda_{C} \simeq 0.23$ the Cabibbo angle, and $\xi$ assumed to be flavor-anarchic with entries of order unity.

CP-odd parameter $\xi$. In general $\xi$ carries some spurious charge under the SM flavor group. It is clear that not all representations are equally viable. For example, if $\xi$ is a SM flavor singlet we anticipate $\bar{\theta} \propto \xi+O\left(y^{2}\right)$, with a proportionality factor controlled by couplings of the unknown dynamics. It is extremely challenging to envision a scenario of this type where $\theta_{\mathrm{CKM}} \sim 1$ and $|\bar{\theta}| \ll 1$. (This is the typical prediction of the earlier literature on spontaneous CP violation.) Similarly, a CP-odd vev in the bi-fundamental or in the sextet has an unsuppressed complex determinant, and would generically result in $\bar{\theta} \propto \operatorname{det}(\xi)$. In these models (1.1) is usually achieved postulating non-trivial cancellations among diagrams. Because we aim to solve the strong CP problem dynamically, we look for better options.

Fortunately there are much promising options. For $\xi$ charged under some flavor representation, $\bar{\theta}$ can only be generated at higher order in $y$, thus providing a rationale behind its smallness. The simplest non-trivial representations of the SM flavor group that have a vanishing $\bar{\theta}$ at leading order in $y$ turn out to be the triplet and octet of $\mathrm{SU}(3)_{Q}$, with $Q=q, u, d$. We will therefore study scenarios with $\xi \sim \mathbf{3}, \mathbf{8} \in \mathrm{SU}(3)_{Q}$. Without any reference to explicit models, we can ask what are the dominant flavor-singlet CP-odd combinations of $y_{u, d}$ and $\xi \sim \mathbf{3}, \mathbf{8} \in \mathrm{SU}(3)_{Q}$ in a general theory. The result is presented in table 1 for flavor-anarchic $\xi$ of order unity. The factors of $4 \pi^{2}$ in the table are estimated using NDA, but one should keep in mind that the actual numerical factors are modeldependent.

Consider first $\xi$ in the adjoint of $\mathrm{SU}(3)_{q}$. In our field basis, under $\mathrm{CP} y_{u, d} \rightarrow y_{u, d}^{*}=y_{u, d}$ are unchanged whereas $\xi \rightarrow \xi^{*}=\xi^{t} \neq \xi$. Note that $\xi$ is a hermitian matrix. Importantly, there is no CP-odd function that involves only the CPV spurion now, because any real function of a hermitian matrix has a real trace. Hence, to generate $\bar{\theta}$ we need insertions of the Yukawa matrices, which play the role of our small expansion parameters, see the second column in table 1.

After integrating out the messenger dynamics of figure 1, the SM Yukawas are

$$
Y_{u, d}=f_{u, d}(\xi) y_{u, d}+O\left(y^{3}\right)
$$


for some function $f_{u, d}$ with real coefficients. A large CKM phase generically follows from $\operatorname{Arg}(\xi) \sim 1$ and $f_{u} \neq f_{d}$. (While intuitively clear, the reader can explicitly check this last statement using for example the results of [40].) Since $\xi \sim \mathbf{8}$ is hermitian, $f_{u, d}$ are as well, and so $\operatorname{det} Y$ is real at $O\left(y^{2}\right)$. At higher order $\theta_{\mathrm{F}, \mathrm{QCD}}$ receive loop corrections. By counting the flavor indices and using the Cayley-Hamilton identity it is easy to see that for $\xi \sim \mathbf{8} \in \mathrm{SU}(3)_{q}$ all flavor-singlets, and $\bar{\theta}$ in particular, can always be written as combinations of traces of polynomials of $y_{u, d}, \xi$ and real determinants, involving unknown real coefficients. ${ }^{4}$

Finally, to estimate the actual size of the invariants in the last column of the table we observe that, because $y=f(\xi) Y+O\left(Y^{3}\right)$, replacing

$$
y \rightarrow Y
$$

results in sub-leading corrections. We can then perform flavor rotations to diagonalize $Y_{u}$ and put the down-type Yukawa in the form $V Y_{d}$ with $Y_{d}$ diagonal and $V$ the CKM matrix. This has no effect on $\bar{\theta}$, which is flavor singlet.

One can proceed analogously for the other representations shown in table 1. Note that for $\xi \sim \mathbf{3} \in \mathrm{SU}(3)_{q}$ one can also find $\mathrm{CP}$-odd expressions such as

$$
\left(y_{u} y_{u}^{\dagger} \xi\right)_{i}\left(y_{d} y_{d}^{\dagger} \xi\right)_{j}\left(y_{u} y_{u}^{\dagger} y_{d} y_{d}^{\dagger} \xi\right)_{k} \epsilon^{i j k}
$$

where we used the fact that $\operatorname{det}\left(y y^{\dagger}\right)$ are real while $A_{i} A_{j} \epsilon^{i j k}=0$ for any real vector $A$. However, the numerical value of (2.4) is smaller than that quoted in the first line of table 1. Furthermore, we will see that in explicit scenarios, flavor triplet $\xi$ s carry another spurious charge under a $\mathrm{U}(1)_{\xi}$, which actually forbids $(2.4)$.

In the case $\xi \sim \mathbf{3} \in \mathrm{SU}(3)_{u}$ we have $\left(f=c_{1}+c_{2} \xi \xi^{\dagger}\right.$, with $c_{1,2}$ real functions of $\left.|\xi|^{2}\right)$

$$
Y_{u}=y_{u} f(\xi)+O\left(y_{u, d}^{3}\right) \quad Y_{d}=y_{d}+O\left(y_{u, d}^{3}\right)
$$

Again, the contributions to $\bar{\theta}$ involving the Levi-Civita tensors are smaller than those of table 1 (i.e. a large number of $y$ s are needed to build a singlet under $\mathrm{U}(1)_{\xi}$ and the axial quark symmetry). Similar considerations hold for $\xi \sim \mathbf{8} \in \mathrm{SU}(3)_{u}$ and $\xi \sim \mathbf{3}, \mathbf{8} \in \mathrm{SU}(3)_{d}$.

Looking at table 1 we conclude that theories with $\xi \sim \mathbf{3} \in \mathrm{SU}(3)_{u}$ or CP-odd spurions charged under $\mathrm{SU}(3)_{d}$ are robustly consistent with data for generic $\xi \sim 1$. In these scenarios the strong CP problem is simply addressed by realizing the framework depicted in figure 1. A class of $4 \mathrm{D}$ theories of this type is discussed in appendix A. In section 3 we will show how to construct alternative 5D realizations that also address the hierarchy problem.

The viability of scenarios with $\xi \sim \mathbf{8} \in \mathrm{SU}(3)_{u}$ is somewhat model-dependent, whereas to make models with $\xi \in \mathrm{SU}(3)_{q}$ compatible with data one should invoke an anti-correlation between $\xi$ and $y_{u, d}$. Because this seems a rather non-generic requirement, we discard $\xi \in \mathrm{SU}(3)_{q}$ in what follows and identify $\xi \in \mathrm{SU}(3)_{u, d}$ as the most promising alternatives.

\footnotetext{
${ }^{4}$ Recall that pairs of Levi-Civita tensors with co-variant anti-covariant indices can be written as traces.
} 


\subsubsection{Generalization}

The above analysis assumes the minimal ingredients (2.2) needed to construct a realistic theory. We now want to study the impact of possible departures from this minimal framework.

First of all, models with multiple unsuppressed and uncorrelated CP-odd spurions with indices in the same $\mathrm{SU}(3)_{Q}$ are disfavored, because in that case $\bar{\theta}$ is typically renormalized at $O\left(y^{2}\right)$, e.g. $\bar{\theta} \propto \operatorname{tr}\left\{y^{\dagger} y\left[f_{1}, f_{2}\right]\right\}$ for $\xi \in \mathrm{SU}(3)_{u, d}$. Similarly, models with $\xi \mathrm{s}$ in different $\mathrm{SU}(3) \mathrm{s}$ are usually disfavored; the only exception are scenarios with one $\xi_{u} \in \mathrm{SU}(3)_{u}$ and one $\xi_{d} \in \mathrm{SU}(3)_{d}$, which are allowed for arbitrary $\xi_{u, d} \sim 1$.

Furthermore, additional flavor-violating CP-even couplings must be small. A modelindependent bound on the latter can be obtained as follows. Denote by $\Lambda_{F}>m_{*}$ the mass threshold at which new $O(1)$ sources of flavor violation arise. Then new contributions to $\bar{\theta}$ will be generated by loops involving the SM, the messengers, and heavy particles of mass $\Lambda_{F}$. We can formally integrate out the CP-invariant flavorful dynamics, obtaining 4-fermion interactions such as $C_{i j k l}\left(\overline{q_{i}} Q_{j}\right)\left(\overline{Q_{k}} q_{l}\right) / \Lambda_{F}^{2}$. Closing a loop with the SM Yukawa, from the latter operators we estimate a 1-loop correction to $Y$ which translates into

$$
\bar{\theta}=\frac{C}{4 \pi^{2}} \frac{m_{*}^{2}}{\Lambda_{F}^{2}} \ln \frac{\Lambda_{F}}{m_{*}} .
$$

For generic $C_{i j k l} \sim 1$ one gets $C \propto Y_{Q_{3}} / Y_{Q_{1}}$, and (1.1) requires $m_{*} / \Lambda_{F} \lesssim 5 \times 10^{-7}$. No parametric suppression is available if $m_{*} \gtrsim \Lambda_{F}$. The lesson we learn is that a robust solution of the strong CP problem via spontaneous CP violation must satisfy $m_{*} / \Lambda_{F} \ll 1$ : flavor anarchy in the messenger sector is severely constrained.

Finally, what about possible flavor-conserving interactions between quarks and the CPV sector? To be conservative, let us ignore the effect of a coupling $\lambda$ to the SM quarks (i.e. take $\xi=0$ ), and assume that the colorless CPV dynamics couples to leptons and/or is charged under the electroweak interactions. Then, it is possible to see that the first Feynman diagrams that can induce a neutron EDM are formally encoded in figure 2 - see the caption for an explanation. We estimate

$$
\bar{\theta} \sim\left(\frac{g_{w}^{2}}{16 \pi^{2}}\right)^{2} \bar{\theta}_{w} \sim 10^{-10}\left(\frac{\bar{\theta}_{w}}{10^{-5}}\right)
$$

where $\bar{\theta}_{w}$ is the electroweak theta angle induced by the CPV sector. We see that the quark flavor-conserving interactions in figure 1 do not represent a serious threat as long as $\left|\bar{\theta}_{w}\right| \lesssim 10^{-5}$. The latter condition is robustly ensured assuming the CPV sector is completely SM neutral, with possible couplings to leptons generated in a way analogous to quarks as discussed above. Yet, it is clear that $\left|\bar{\theta}_{w}\right| \lesssim 10^{-5}$ can be accommodated in other ways. ${ }^{5}$

\footnotetext{
${ }^{5}$ We stress that our conclusions do not obviously generalize to scenarios with a non-minimal electroweak symmetry-breaking sector, where $\bar{\theta}$ might be induced by the complex vev of exotic Higgs fields, see e.g. $[42,43]$. Ultimately, the reason is that in those models CP violation is controlled by a flavor-singlet $\xi$, a possibility that we want to avoid.
} 


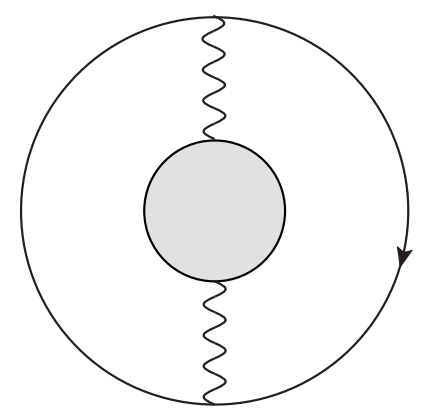

Figure 2. This diagram illustrates how a CP-violating non-colored sector can contribute to $\bar{\theta}$ via gauge loops. The grey blob refers to loops of colorless particles, the wavy lines are gauge bosons, and the outer solid line is a colored fermion (quark or messenger). By cutting the external solid line and attaching a Higgs or a mass if necessary, as well as an external $\xi$ to the blob, the diagram renormalizes the colored fermion mass, and hence $\bar{\theta}$. Alternatively, by attaching gluons to the solid line, the graph becomes a direct contribution to $\theta_{\mathrm{QCD}}$ (see, e.g., [41]).

\section{A realistic model on $\mathrm{AdS}_{5}$}

As already stressed, the relation (2.1) is basically the requirement that quarks mix at $O(1)$ with some exotic messenger sector. The problem of constructing theories of this type without tuning is therefore analogous to obtaining a large top mass in models with $\mathrm{TeV}$ scale compositeness. We illustrate this by constructing a realistic 5D model that also addresses the Planck-TeV hierarchy as in [44, 45]. Furthermore, the existence of an approximate SM flavor symmetry ensures that this scenario is an ideal realization of our scheme, see figure 1.

\subsection{Setup}

The basic ingredients needed to meet the criteria of figure 1 are minimal flavor violation (MFV) violated by the CP-odd (and flavor-violating) spurion $\xi$. There are several ways in which this can be realized on a warped extra dimension. MFV can be obtained as discussed using a CFT language in [46]. We focus on a scenario with composite $u, d$, of which the $5 \mathrm{D}$ realization has not been explicitly presented yet. Models with composite $q$ s or a fully composite SM are also possible, see e.g. [47, 48], but more constrained by electroweak data.

Consider a slice of $\mathrm{AdS}_{5}$

$$
d s^{2}=a^{2}\left(\eta_{\mu \nu} d x^{\mu} d x^{\nu}-d z^{2}\right) \quad a=\frac{L}{z},
$$

in the interval $z \in\left[z_{\mathrm{UV}}, z_{\mathrm{IR}}\right]$. Below we will see that the size of the extra dimension is stabilized such that $1 / z_{\mathrm{IR}} \sim m_{*} \sim \mathrm{TeV}$ with $1 / z_{\mathrm{UV}} \sim \Lambda \sim 10^{18} \mathrm{GeV}$. The bulk and the IR brane respect $\mathrm{CP}$ and the following gauge symmetry

$$
\mathrm{SU}(3)_{C} \times \mathrm{SU}(2)_{w} \times \mathrm{U}(1)_{Y} \times G_{F} \times \mathrm{U}(1)_{\xi},
$$

where $G_{F}=\mathrm{SU}(3)_{u} \times \mathrm{SU}(3)_{d}$. It is straightforward to enlarge the gauge symmetry to account for a custodial $\mathrm{SU}(2)$. 
The UV brane violates $G_{F}$ at $O(1)$. On the other hand, $\mathrm{CP} \times \mathrm{U}(1)_{\xi}$ are spontaneously broken by a UV-localized scalar $\Sigma$ with charge $+1 / 2$ under $\mathrm{U}(1)_{\xi}$, and a vev set by a scale $m_{\mathrm{CP}} \ll \Lambda$. This can be naturally achieved by promoting $\Sigma$ to a bulk scalar of another $\mathrm{AdS}_{5}$ throat, or (equivalently) by identifying $\Sigma$ with a fermion bilinear, as shown in appendix C. The additional requirement $m_{\mathrm{CP}} \gg T_{\mathrm{RH}} \gg 1 / z_{\mathrm{IR}}$, with $T_{\mathrm{RH}}$ the re-heating temperature, guarantees that a potential domain wall problem associated to the spontaneous breaking of $\mathrm{CP}$ is evaded. This requirement motivates our choice of localizing $\Sigma$ on the UV, rather than in the bulk, though we emphasize that the strong CP problem may still be solved with a bulk $\Sigma$.

The CP-odd scalar has very suppressed couplings to the SM fermions, that are $\mathrm{U}(1)_{\xi}$ neutral (see below). CP violation shines through the bulk thanks to the UV-localized coupling

$$
\left.\lambda \Sigma \phi\right|_{z_{\mathrm{UV}}}
$$

with $\phi$ a bulk scalar in the fundamental representation of $\mathrm{SU}(3)_{u}$ and transforming with charge $-1 / 2$ under $\mathrm{U}(1)_{\xi}$. We will show that this will lead to $\xi \propto \lambda\langle\Sigma\rangle \sim \mathbf{3} \in \mathrm{SU}(3)_{u}$.

\subsection{Minimal flavor and CP violation}

There are 4 types of bulk fermions: ${ }^{6} \psi_{u, d}$, that have the same SM charges of the righthanded quarks $u, d$, and two weak doublets $\psi_{q_{u}, q_{d}}$ with the same SM charges as $q$. They transform under $G_{F}$ as $\psi_{u, q_{u}} \sim \mathbf{3} \in \mathrm{SU}(3)_{u}, \psi_{d, q_{d}} \sim \mathbf{3} \in \mathrm{SU}(3)_{d}$. All fermions are $\mathrm{U}(1)_{\xi^{-}}$ singlets.

The boundary conditions are

$$
z_{\mathrm{IR}}\left\{\begin{array} { l } 
{ \psi _ { u , d } | _ { L } = 0 } \\
{ \psi _ { q _ { u } , q _ { d } } | _ { R } = 0 }
\end{array} \quad z _ { \mathrm { UV } } \left\{\begin{array}{l}
\left.\psi_{u, d}\right|_{L}=0 \\
\left.\psi_{q_{u}}\right|_{L}=\left.F \psi_{q_{d}}\right|_{L}
\end{array}\right.\right.
$$

where $F$ is a 3 by $3 G_{F}$-violating matrix, real by CP. The SM $u, d$ arise as the zero modes of $\psi_{u, d}$, whereas a single massless left-handed combination of the doublets survives (3.4) and will be identified with $q$.

The $\left(G_{F}\right.$-symmetric) bulk fermion masses are chosen so that the zero-modes of $\psi_{u, d}$ peak in the IR, whereas $\psi_{q_{u}, q_{d}}$ are localized towards the UV. In terms of the bulk mass parameters $c=m_{5} L$ this reads $c_{u, d}<1 / 2, c_{q_{u}, q_{d}}>1 / 2$.

Consistently with our assumptions we add $G_{F}$-violating UV-localized kinetic terms for the fermions

$$
\mathcal{L}_{\text {kin }}=\sum_{Q=u, d, q_{u}, q_{d}} \int d z a^{4}(z) \overline{\psi_{Q}} K_{Q} i \not D \psi_{Q} \delta\left(z-z_{\mathrm{UV}}\right),
$$

with $K_{Q}$ symmetric (real) matrices, as well as a UV mass for the $G_{F}$ gauge field, which ensures that the low energy theory has no exotic massless vectors.

\footnotetext{
${ }^{6}$ With this field content, there are $G_{F}^{3}, G_{F}^{2} \times \mathrm{U}(1)_{Y}$ anomalies, which we assume are cancelled by UVlocalized fermions. These acquire masses of order $\Lambda$ after $G_{F}$ breaking, and have no phenomenological impact.
} 
The kinetic terms affect the normalization of the $4 \mathrm{D}$ modes. However, because the heavy modes are localized towards the IR brane, the effect of the UV kinetic terms on them is suppressed by powers of $z_{\mathrm{UV}} / z_{\mathrm{IR}}$ and is numerically negligible. Similar considerations hold for the zero-modes of $\psi_{u, d}$. It follows that $G_{F}$-violation is entirely encoded in the UV kinetic terms and UV boundary conditions of the would-be zero-modes of $\psi_{q_{u}, q_{d}}$.

The wave-functions of the left-handed zero-mode reads

$$
\psi_{q_{u}}^{0}=\frac{1}{\sqrt{L}}\left(\frac{z}{z_{\mathrm{UV}}}\right)^{2-c_{q_{u}}} F N q(x) \quad \psi_{q_{d}}^{0}=\frac{1}{\sqrt{L}}\left(\frac{z}{z_{\mathrm{UV}}}\right)^{2-c_{q_{d}}} N q(x),
$$

where the normalization $N$ is a matrix in flavor space that satisfies

$$
1=N^{\dagger}\left[\mathcal{K}_{q_{d}}+F^{\dagger} \mathcal{K}_{q_{u}} F\right] N, \quad \mathcal{K}_{Q} \equiv a^{4}\left(z_{\mathrm{UV}}\right)\left[\frac{z_{\mathrm{UV}}}{L} \frac{\left(\frac{z_{\mathrm{IR}}}{z_{\mathrm{UV}}}\right)^{1-2 c_{Q}}-1}{1-2 c_{Q}}+K_{Q}\right] .
$$

This guarantees that $q$ has a canonical kinetic term in the effective $4 \mathrm{D}$ theory. (We neglected IR kinetic terms, that are flavor-diagonal and irrelevant to our discussion.)

In the effective field theory, flavor-violation is controlled by the two matrices $F N, N$, which may be interpreted as spurions transforming respectively as a $(\overline{\mathbf{3}}, \mathbf{3}, \mathbf{1})$ and $(\overline{\mathbf{3}}, \mathbf{1}, \mathbf{3})$ under $\mathrm{SU}(3)_{q} \times \mathrm{SU}(3)_{u} \times \mathrm{SU}(3)_{d}$. The resulting model satisfies minimal flavor (and CP) violation. In particular, the SM Yukawa couplings arise from the IR-localized operators $Y_{u *} L \overline{\psi_{q_{u}}} \widetilde{H} \psi_{u}+Y_{d *} L \overline{\psi_{q_{d}}} H \psi_{d}$, with $Y_{u *, d *}$ real numbers, and after KK reduction the protoSM Yukawa matrices are

$$
\begin{aligned}
& y_{u}=Y_{u *} N^{\dagger} F^{\dagger} \sqrt{1-2 c_{u}}\left(\frac{L}{z_{\mathrm{UV}}}\right)^{3 / 2}\left(\frac{z_{\mathrm{UV}}}{z_{\mathrm{IR}}}\right)^{c_{q_{u}}-1 / 2} \\
& y_{d}=Y_{d *} N^{\dagger} \sqrt{1-2 c_{d}}\left(\frac{L}{z_{\mathrm{UV}}}\right)^{3 / 2}\left(\frac{z_{\mathrm{UV}}}{z_{\mathrm{IR}}}\right)^{c_{q_{d}}-1 / 2}
\end{aligned}
$$

up to hierarchy-suppressed corrections. Here for simplicity we assumed the Higgs boson is IR-localized, but could have considered a bulk scalar or a Nambu-Goldstone Higgs. Furthermore, note that because of the suppression $\left(z_{\mathrm{UV}} / z_{\mathrm{IR}}\right)^{c-1 / 2}$, realistic quark masses can be obtained with $K_{Q}=O(1)$ (though no explanation of the hierarchy is offered by our setup).

\section{3 $\quad \theta_{\mathrm{CKM}} \sim 1$, dynamically}

We anticipated that $\mathrm{CP}$ is violated on the UV and shined through the bulk using a bulk scalar $\phi \sim \mathbf{3} \in \mathrm{SU}(3)_{u}$. The Lagrangian of $\phi$ reads

$$
\begin{aligned}
\mathcal{L}_{\phi}= & \int d z a^{3}\left[\eta^{\mu \nu} D_{\mu} \phi^{\dagger} D_{\nu} \phi-D_{5} \phi^{\dagger} D_{5} \phi-a^{2} m_{\phi}^{2} \phi^{\dagger} \phi+\cdots\right] \\
& +\left.a^{4}\left[-\frac{T_{\mathrm{UV}}}{L^{4}}+\left(\frac{J^{\dagger}}{L^{5 / 2}} \phi+\mathrm{hc}\right)+\cdots\right]\right|_{z_{\mathrm{UV}}} \\
& +\left.a^{4}\left[-\frac{T_{\mathrm{IR}}}{L^{4}}-\frac{m_{\mathrm{IR}}}{L} \phi^{\dagger} \phi+\cdots\right]\right|_{z_{\mathrm{IR}}}
\end{aligned}
$$


where $\cdots$ refer to higher order couplings. The non-linear bulk couplings of $\phi$ are assumed to be somewhat suppressed, while the boundary terms have generic coefficients (this is possible by locality of the $5 \mathrm{D}$ theory).

Because $m_{\phi}^{2}>-4$, higher-dimensional operators on the UV brane are naturally suppressed by powers of the hierarchy and can be neglected. The dominant UV interaction is the source term, that encodes the coupling to the UV-localized CP-violating sector (see $(3.3))$

$$
J^{\dagger}=L^{5 / 2} \lambda\langle\Sigma\rangle
$$

As shown in appendix $\mathrm{C}$, the dynamics of $\Sigma$ is completely irrelevant to us, only the vacuum $\langle\Sigma\rangle$ has phenomenological relevance. In what follows we will treat $J$ as a dimensionless complex constant with $|J| \ll 1$.

The source $J$ induces a complex vacuum expectation value for $\phi$, which eventually feeds into $\theta_{\mathrm{CKM}}, \bar{\theta}$. Imposing Neumann conditions on the IR we find, up to $O\left(1 / M_{5}^{3}\right)$ with $M_{5}$ the 5D Planck scale, the following profile

$$
\phi(z)=\frac{J}{\epsilon L^{3 / 2}}\left(\frac{z}{z_{\mathrm{UV}}}\right)^{-\epsilon} \frac{1-\eta\left(\frac{z}{z_{\mathrm{IR}}}\right)^{4+2 \epsilon}}{1+\frac{4+\epsilon}{\epsilon} \eta\left(\frac{z_{\mathrm{UV}}}{z_{\mathrm{IR}}}\right)^{4+2 \epsilon}}, \quad \eta=\frac{m_{\mathrm{IR}}-\epsilon}{4+m_{\mathrm{IR}}+\epsilon}
$$

where $\epsilon=-2+\sqrt{4+m_{\phi}^{2} L^{2}}$.

To communicate CP violation to the SM we need to couple $\phi$ to the SM quarks. A simple option is to add a messenger fermion $\Psi$ in an appropriate representation of the gauge group to allow a trilinear coupling among $\phi, \Psi$ and $\psi_{u}$. The boundary conditions for $\Psi$ are chosen so that there are no zero modes. Integrating out $\Psi$ one gets a correction to the kinetic term of $\psi_{u}$ of order $\xi \xi^{\dagger}$, where $\xi \sim \lambda_{*} \phi\left(z_{\mathrm{IR}}\right) L^{3 / 2} / m_{\Psi} z_{\mathrm{IR}}$, with $\lambda_{*}$ the dimensionless trilinear coupling. After having canonically normalized the quark $u$ this results in a complex Yukawa. Since $m_{\Psi} \sim \pi / z_{\mathrm{IR}}$, we see that $\theta_{\mathrm{CKM}} \sim 1$ is obtained for $\phi\left(z_{\mathrm{IR}}\right) L^{3 / 2}=O(1)$, at least as long as $\lambda_{*}$ is not too small (for large $\lambda_{*}$ the CKM phase is effectively generated by higher dimensional operators, as discussed below). This mechanism is analogous to that of [18-20] (see appendix A), although here $\theta_{\mathrm{CKM}} \sim 1$ is dynamically generated.

One can envision other couplings between $\phi$ and the SM quarks that ensure $\theta_{\text {CKM }} \sim 1$ equally well. This shows that in general these models are not in the Nelson-Barr class. A large CKM phase may be parametrized in a model-independent way by IR-localized higher dimensional operators such as

$$
c_{\mathrm{IR}_{u, d}} \frac{16 \pi^{2}}{\Lambda_{5}^{4}} \overline{\psi_{q_{u}, u}} \phi \phi^{\dagger} i \not D \psi_{q_{u}, u}, \quad c_{Y_{u}} \frac{\left(16 \pi^{2}\right)^{3 / 2}}{\Lambda_{5}^{4}} \overline{\psi_{q_{u}}} \phi \phi^{\dagger} \widetilde{H} \psi_{u}
$$

where according to NDA $c_{\mathrm{IR}_{u, d}, Y_{u}}<1$. The $5 \mathrm{D}$ cutoff can be written as $\Lambda_{5}=N_{\mathrm{KK}} \frac{\pi}{L}$, where $N_{\mathrm{KK}}$ is a measure of the number of weakly coupled KK modes that can be described by our effective theory. Inserting the vev of $\phi$ one finds a correction to the Yukawa coupling of the form anticipated in (2.5) with $\xi \xi^{\dagger} \propto \phi\left(z_{\mathrm{IR}}\right) \phi^{\dagger}\left(z_{\mathrm{IR}}\right) / \Lambda_{5}^{4} L$. We now need $\phi\left(z_{\mathrm{IR}}\right) L^{3 / 2} \sim$ $5\left(N_{\mathrm{KK}} / 5\right)^{2}$ to generate a large CKM phase. 
We conclude that $\theta_{\mathrm{CKM}} \sim 1$ generically follows provided $\phi\left(z_{\mathrm{IR}}\right) L^{3 / 2}=O(1)$, which via (3.10) represents a non-trivial relation between $|J| \ll 1$ and $z_{\mathrm{UV}} / z_{\mathrm{IR}} \ll 1$. Importantly, a nice feature of these 5D models is that such relation can be naturally achieved if $\phi$ is responsible for stabilizing the extra dimension. Following $[45,47]$ we determine the size of the extra dimension by minimizing the potential $\mathcal{V}_{\text {eff }}$ of $\mu=1 / z_{\mathrm{IR}}$. Inserting (3.10) into (3.9) we get:

$$
\begin{aligned}
\mathcal{V}_{\text {eff }}(\mu) & \equiv-\left.\mathcal{L}_{\phi}\right|_{\text {on-shell }} \\
& =\mu^{4}\left[T_{\mathrm{IR}}+\frac{(4+2 \epsilon)}{\epsilon^{2}} \eta J^{\dagger} J\left(\frac{\mu}{\mu_{0}}\right)^{2 \epsilon}\right]+\mu_{0}^{4}\left[T_{\mathrm{UV}}-\frac{J^{\dagger} J}{\epsilon}\right]+O\left(\left(\mu / \mu_{0}\right)^{8+4 \epsilon}\right),
\end{aligned}
$$

where $\mu_{0}=1 / z_{\mathrm{UV}}$. In our model the operator dual to $\phi$ is relevant (i.e. $\epsilon<0$ ) and $|\epsilon|<1$, so a stable vacuum requires $T_{\mathrm{IR}}>0$ and $\eta<0$ (which is equivalent to $-4-\epsilon<m_{\mathrm{IR}}<\epsilon$ ). The minimum, up to $O\left(\left(\mu / \mu_{0}\right)^{4+2 \epsilon}\right)$, is determined by:

$$
\frac{J^{\dagger} J}{\epsilon^{2}}\left(\frac{\mu}{\mu_{0}}\right)^{2 \epsilon}=\frac{\phi^{2}\left(z_{\mathrm{IR}}\right) L^{3}}{(1-\eta)^{2}}=-\frac{4}{(4+2 \epsilon)^{2}} \frac{T_{\mathrm{IR}}}{\eta} .
$$

As anticipated, we see that $\phi\left(z_{\mathrm{IR}}\right) L^{3 / 2}=O(1)$ is obtained for $T_{\mathrm{IR}}, \eta \sim 1$. From this $\theta_{\mathrm{CKM}}=O(1)$ automatically follows.

What we have just reviewed is the familiar Goldberg-Wise mechanism [45, 47] with a naturally small (by the $\mathrm{U}(1)_{\xi}$ ) UV source, and a relatively large $|\epsilon|$, say $\sim 0.3-0.4$. The effective potential $\mathcal{V}_{\text {eff }}$ was calculated ignoring gravity fluctuations, which are down by powers of $\phi^{2} / M_{5}^{3} \lesssim 1 /\left(M_{5} L\right)^{3}$. We explicitly checked that our conclusion $\xi \sim 1$ is not spoiled for $\phi^{2} \sim M_{5}^{3}$, nor for generic IR-localized $\phi$ potentials. The reason may be ultimately understood using the CFT language of the next subsection. ${ }^{7}$

\subsection{CFT interpretation and estimate of $\bar{\theta}$}

To show how robust the solution of the strong CP problem is, it is instructive to estimate $\bar{\theta}$ using a general CFT language.

The models introduced in this section (as well as those of appendix A) belong to a larger class of scenarios realizing the framework of figure 1, that are described by a (large $N)$ CFT with global symmetry $\supset \mathrm{SU}(3)_{u, d}$. The CFT is deformed by the couplings:

$$
\delta \mathcal{L}_{\mathrm{CFT}}=y_{u} O_{u}+y_{d} O_{d}+\lambda \Sigma O
$$

In our model the global symmetry is (3.2), $O_{u, d}=\bar{q} \mathcal{O}_{u, d}$, with $\mathcal{O}_{u, d}$ fermionic operators of the CFT with scaling dimension $2+c_{q_{u}, q_{d}}>5 / 2$, whereas $O$ is a scalar of dimension $d_{O}=$ $4+\epsilon<4$ dual to $\phi$. Higher dimensional operators, and loops of the CP-violating dynamics,

\footnotetext{
${ }^{7}$ As usual, the scalar spectrum of the model contains a tower of heavy modes of mass $\sim \pi / z_{\mathrm{IR}}$ and a light radion. In the limit of small back-reaction the latter can be efficiently interpolated by $\mu$ (see for instance [49]). Defining the 5D Einstein-Hilbert action by $\int \sqrt{|g|}\left[-M_{5}^{3} R\right]$, and identifying $z_{\mathrm{UV}}=L$, we find that the kinetically normalized radion is $\sqrt{6\left(M_{5} L\right)^{3}} \mu$, and $m_{\mathrm{rad}}^{2}=\frac{4}{3} \frac{T_{\mathrm{IR}}}{\left(M_{5} L\right)^{3}} \frac{|\epsilon|}{z_{\mathrm{IR}}^{2}}$.
} 
can be rendered completely negligible under very generic conditions on $m_{\mathrm{CP}} / \Lambda, d_{O}, d_{\Sigma}$, with $d_{\Sigma}$ the scaling dimension of $\Sigma$ (see appendix C).

To generate a large CKM phase dynamically, as achieved in section 3.3, $O$ must be the most relevant deformation of the $\mathrm{CFT}, d_{O}<4$. After symmetry breaking, the CP-violating coupling $\lambda\langle\Sigma\rangle$ grows towards the IR until the scale $m_{*}$ defined by

$$
\xi \equiv J\left(\frac{m_{*}}{\Lambda}\right)^{d_{O}-4} \sim 1
$$

with $J \equiv \lambda\left(m_{\mathrm{CP}} / \Lambda\right)^{d_{\Sigma}}$. This is the CFT dual of (3.13), with $m_{*} \sim 1 / z_{\mathrm{IR}}$. Note that $\xi$ has the same spurious charges as $O$ under $G_{F}$ rotations. Below $m_{*}, \mathrm{CP}$ violation is controlled by $\xi$, and a large CKM phase is generated provided the CFT is sufficiently generic.

The setup of figure 1 is realized and, according to the results of section 2, we expect $\bar{\theta}$ will be under control choosing appropriate charges for $O$ (in the above 5D model $O \sim$ $\left.\xi \sim \mathbf{3} \in \mathrm{SU}(3)_{u}\right)$. Importantly, the expansion parameter here is truly the mixing $y^{2} / g_{*}^{2}$ between $q$ and the heavy resonances (KK modes) — with $g_{*}$ the typical coupling among resonances of the CFT - and can be much larger than $y^{2} / 4 \pi^{2}$, which we would find in a renormalizable $4 \mathrm{D}$ model.

In our model $\bar{\theta}$ cannot arise at tree-level. Tree-level corrections to $\operatorname{det}(Y)$ are written as traces of polynomials of $y_{u} D_{u} y_{u}^{\dagger}, y_{d} D_{d} y_{d}^{\dagger}$, where $D_{u}$ (a function of $\xi \xi^{\dagger}$ ) and $D_{d} \propto 1$ are the propagators of $\mathcal{O}_{u}$ and $\mathcal{O}_{d}$, but these do not renormalize $\bar{\theta}$ because the polynomials are symmetric under the exchange of the building blocks. To get $\bar{\theta}$ one needs insertions of 4-point functions involving $\mathcal{O}_{u, d}$, which suppress the amplitude by at least a factor of $g_{*}^{2} / 4 \pi^{2}$. Actually, neglecting higher-dimensional operators, in our 5D model the first contribution to $\bar{\theta}$ arises at 2-loop order via $\Psi \phi \psi_{u}$ and the Yukawa coupling, and is therefore further suppressed by a factor $g_{*}^{2} / 4 \pi^{2}$ compared to the generic CFT case. This gives $\bar{\theta} \sim 10^{-11}\left(1 / g_{*}\right)^{2}$, implying that these theories are capable of solving the strong CP problem even in the large $N$ regime $g_{*} \ll 4 \pi$. Consistently, a similar estimate is obtained when turning on higher-dimensional operators. Diagrammatically, the loop effects can be seen as corrections to $\theta_{\mathrm{F}}$ or direct contributions to $\theta_{\mathrm{QCD}}[41]$.

\section{Phenomenology}

In this section we discuss signatures that, as opposed to $\bar{\theta}$, decouple with the new physics scale $m_{*}$.

\subsection{Electroweak observables}

Models with spontaneous $\mathrm{CP}$ violation require a large mixing between right-handed quarks and a messenger sector, see (2.1). In weakly-coupled $4 \mathrm{D}$ realizations (see appendix A), after having rotated away the $Q / \Psi$ mixing, the relevant interactions are controlled by $\bar{q} \widetilde{H} \Psi$ for $\xi \in \mathrm{SU}(3)_{u}$ (or $\bar{q} H \Psi$ for $\xi \in \mathrm{SU}(3)_{d}$ ) with coupling of order $Y_{u} / m_{*}$ (or $Y_{d} / m_{*}$ ). Here $m_{*}^{2}=m_{\Psi}^{2}+|\lambda \Sigma|^{2}$ is the mass of the messenger up to small corrections of order $m_{t, b}^{2} / m_{*}^{2} \ll 1$, see appendix A for details. Contributions to the electroweak observables arise at 1-loop. Using the results of, e.g., $[50,51]$ we find that the effect is well within 
current bounds $[52,53]$ as soon as $m_{t} / m_{*} \lesssim 0.1$ (for $\left.\xi \in \mathrm{SU}(3)_{u}\right)$ and $m_{b} / m_{*} \lesssim 0.04$ (for $\left.\xi \in \mathrm{SU}(3)_{d}\right)$. This translates into a bound $m_{*} \gtrsim 1.5 \mathrm{TeV}$ for our top-partners, whereas a negligible constraint on partners of the bottom quark.

In the $5 \mathrm{D}$ models, the entire bulk plays the role of the messenger dynamics. As is well know, warped 5D scenarios are subject to severe bounds from electroweak data. For example, writing the $S$ parameter as $\Delta S=8 \pi v^{2} / m_{*}^{2}$ and conservatively imposing $\Delta S \lesssim 0.2[52,53]$, we find $m_{*} \gtrsim 2.8 \mathrm{TeV}$. The other electroweak constraints do not represent a major concern for the specific model discussed here. For example, the electroweak $T$ parameter can be made compatible with data imposing a custodial symmetry in the bulk. On the other hand, stronger constraints arise from $Z^{0}$ pole observables on models with "composite doublets", $\xi \in \mathrm{SU}(3)_{q}[46]$.

\subsection{Flavor violation}

Flavor violation is a generic implication of our scenarios, with the dominant effects beyond MFV controlled by $\xi$. In the $4 \mathrm{D}$ models discussed in appendix $\mathrm{A}, \Delta F=2$ transitions are controlled by a loop-induced $\left(\bar{q} \gamma^{\mu} Y Y^{\dagger} q\right)^{2}$, and current Kaon data are satisfied for $m_{*} \gtrsim 1 \mathrm{TeV}$ [54-56], even in the worst case scenario $\xi \sim \mathbf{3} \in \mathrm{SU}(3)_{u} . B \rightarrow X_{s, d}$ transitions are also relevant. Considering again the more constrained scenario $\xi \sim \mathbf{3} \in \mathrm{SU}(3)_{u}$, those are controlled by

$$
\overline{d_{L}}\left[V^{\dagger} Y_{u} f Y_{u}^{\dagger} V Y_{d}\right] d_{R}
$$

Using standard results (see e.g. [57]), we find that the main effect is described by the operator $Q_{7 \gamma}=\frac{G_{F}}{\sqrt{2}} V_{t s}^{*} V_{t b} \frac{e}{4 \pi^{2}} m_{b} \overline{s_{L}} \gamma^{\mu \nu} b_{R} F_{\mu \nu}$, with a coefficient $\delta C_{7 \gamma}\left(m_{*}\right) \sim m_{t}^{2} /\left(3 m_{*}^{2} V_{t s}^{*} V_{t b}\right)$. Conservatively requiring $\left|\delta C_{7 \gamma}(3 \mathrm{GeV})\right| \lesssim 0.1$ sets again a bound on the messenger scale of order $m_{*} \gtrsim 1 \mathrm{TeV}$.

We now discuss flavor-violation in $5 \mathrm{D}$ models with $\xi \in \mathrm{SU}(3)_{u}$, since scenarios with $\xi$ charged under $\mathrm{SU}(3)_{d}$ are now subject to more severe constraints from Kaon physics. Flavor-violating amplitudes in $5 \mathrm{D}$ models with $\xi \sim \mathbf{3}, \mathbf{8} \in \mathrm{SU}(3)_{u}$ can be written as polynomials with real coefficients of:

$$
Y_{d}, \quad V^{\dagger} Y_{u}, \quad \xi
$$

where $Y_{u, d}$ are diagonal SM Yukawas (with negligible $\bar{\theta}$ ) and $V$ the CKM matrix. The analysis is a slight generalization of that in [58]. For example, all bounds on $\Delta F=1,2$ processes involving $q, d$ derived there immediately apply here as well. The exception are operators involving up type quarks. The most severely constrained is by far the $\Delta F=2$ interaction:

$$
\frac{g_{*}^{2}}{m_{*}^{2}}\left(\overline{u_{R}} \gamma^{\mu} c_{R}\right)^{2}
$$

These have unsuppressed Wilson coefficients in a generic dynamics with anarchic $\xi$. A conservative bound from $D^{0}-\overline{D^{0}}$ mixing gives $m_{*} \gtrsim g_{*} \times 10^{3} \mathrm{TeV}$ [54-56]. 


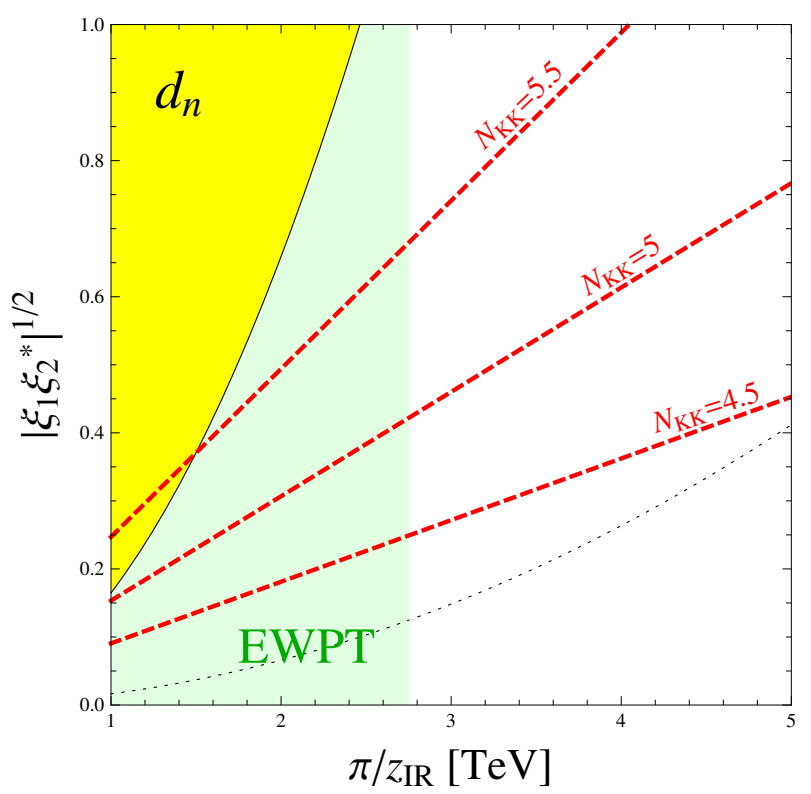

Figure 3. Constraints from $d_{n}$, electroweak, and flavor data in 5D models with $\xi \sim \mathbf{3} \in \mathrm{SU}(3)_{u}$. The contribution of the dim- 6 operators to $d_{n}$ is dominated by the second term in (4.4). We took $\left|b_{u}\left(f_{3}\right)_{31}\right|=\left|\xi_{1}\right|$ and $d_{n}=\operatorname{Im}\left(d_{u}\right)_{11}$, with $\xi_{i}$ defined in the basis with diagonal $Y_{u}$. The yellow area is excluded by current bounds, whereas the dotted black line shows the limit for a constraint 10 times stronger. An indicative measure of the electroweak constraints on $\pi / z_{\mathrm{IR}}$ is shown by the light green area. A conservative bound from flavor physics can be obtained assuming that the IR-localized operator $\left(\overline{\psi_{u}} \gamma^{\mu} \phi \phi^{\dagger} \psi_{u}\right)^{2}$ is generated at the 5D cutoff with a coefficient of order $\left(16 \pi^{2}\right)^{3} / \Lambda_{5}^{10}$, which is the maximal value allowed by calculability. The parameter space excluded by imposing the constraint from $D^{0}-\overline{D^{0}}$ is above the dashed red lines, obtained with $\Lambda_{5} L=N_{\mathrm{KK}} \pi$ and $\phi^{2}\left(z_{\mathrm{IR}}\right) L^{3}=1$.

A quantitative estimate of the bound can be obtained for the scenario of section 3 . In that case (4.3) receives corrections at tree-level by the $G_{F}$ gauge bosons, that have nonuniversal masses $\propto \xi$. However, these can be suppressed by simply assuming a small 5D gauge coupling. A reasonable expectation is that (4.3) is dominantly generated by physics above the cutoff $\Lambda_{5}$. The leading IR-localized operators (recall that $\phi$ is suppressed in the bulk) for $\xi \sim \mathbf{3} \in \mathrm{SU}(3)_{u}$ schematically look like $\left(\overline{\psi_{u}} \gamma^{\mu} \phi \phi^{\dagger} \psi_{u}\right)^{2}$. Because of the large engineering dimension, these can easily be under control if $\phi L^{3 / 2} \sim 1$ and KK masses not far above the $\mathrm{TeV}$, see figure 3. Yet, for $\phi L^{3 / 2} \sim 1$ also the higher-dimensional interactions (3.11) are suppressed, and $\theta_{\mathrm{CKM}}$ must be generated by the tree-level exchange of a messenger $\Psi$ as explained above (3.11).

Alternatively, we may relax the assumption of flavor anarchy for $\xi$. After all, flavor violation is controlled by unknown UV physics which must generate hierarchical SM Yukawas. It does not seem un-reasonable that $\xi$ is also hierarchical in the same field basis in which the $Y_{u, d}$ are. For example, a structure like $\left(y_{u, d}\right)_{i j} \sim \epsilon_{i}^{q} \epsilon_{j}^{u, d}$ and $\xi_{i} \sim \epsilon_{i}^{u}$, with hierarchical $\epsilon \mathrm{s}$, can still generate $\theta_{\mathrm{CKM}} \sim 1$ but dramatically relaxes the bounds on (4.3). On the other hand, the estimates in table 1 depend on the mixing between the second and third generations, and are therefore unaffected. In this class of models $t \rightarrow c$ transitions are a key signature, and $\theta_{\text {CKM }}$ may well be generated by the higher dimensional operators in (3.11). 


\subsection{Neutron EDM from dimension-6 operators}

In addition to $\bar{\theta}$, there are higher dimensional operators that contribute to the neutron EDM, and can easily dominate. ${ }^{8}$ At dimension six the most relevant are $G G \widetilde{G}, H^{\dagger} H G \widetilde{G}$, quark dipoles, and 4-fermion interactions. The first class of operators is flavor-conserving, very much like $G \widetilde{G}$, so its effect is suppressed by $\sim \Lambda_{\mathrm{QCD}}^{2} / m_{*}^{2}$ compared to $\bar{\theta}$ and can be neglected.

The bounds on the dipoles are model-dependent. We start with $\xi \sim \mathbf{3}, \mathbf{8} \in \mathrm{SU}(3)_{u}$ and consider $d_{u} \bar{q} \widetilde{H} \sigma^{\mu \nu} u F_{\mu \nu}$. We use $d_{n} \sim d_{u, d}$ to conservatively estimate the bounds. In realizations of the setup of figure 1, the neutron EDM is of order (the structure $\left(Y_{u} f\right)_{i i}$ has no imaginary part because the $f_{\mathrm{s}}$ are hermitian)

$$
\begin{aligned}
\operatorname{Im}\left(d_{u}\right)_{11} & =\frac{e}{4 \pi^{2}} \frac{v}{m_{*}^{2}} \operatorname{Im}\left[a_{u} Y_{u} f_{1} Y_{u}^{\dagger} Y_{u} f_{2}+b_{u} V Y_{d} Y_{d}^{\dagger} V^{\dagger} Y_{u} f_{3}+O\left(Y^{5}\right)\right]_{11} \\
& =a_{u} e \frac{m_{u}}{m_{*}^{2}} \frac{Y_{u_{k}}^{2}}{4 \pi^{2}} \operatorname{Im}\left[\left(f_{1}\right)_{1 k}\left(f_{2}^{*}\right)_{1 k}\right]+b_{u} e \frac{m_{u_{i}}}{m_{*}^{2}} \frac{Y_{d_{k}}^{2}}{4 \pi^{2}} \operatorname{Im}\left[V_{1 k} V_{i k}^{*}\left(f_{3}\right)_{i 1}\right]+O\left(Y^{5}\right),
\end{aligned}
$$

where $a_{u}, b_{u}$ are model-dependent real numbers $\sim 1$ and the $f$ s polynomials of $\xi$ with real coefficients. For flavor-anarchic $f$ s of order unity, the $90 \%$ CL bound $\left|d_{n}\right| / e<2.9 \times 10^{-26}$ $\mathrm{cm}$ [1] becomes $m_{*} \gtrsim \sqrt{\left|a_{u}\right|} 3.7, \sqrt{\left|b_{u}\right|} 2.5 \mathrm{TeV}$. Yet, in the $4 \mathrm{D}$ models of appendix A we find $f_{1} \propto \xi \xi^{\dagger}, f_{2,3} \propto 1_{3 \times 3}$, and the bound is negligible. The dipole for the down quark leads to similar results.

Similar considerations hold for $\xi \sim \mathbf{3}, \mathbf{8} \in \mathrm{SU}(3)_{d}$ if we replace $Y_{u} \leftrightarrow V Y_{d}$ in (4.4), and we find $m_{*} \gtrsim \sqrt{\left|a_{d}\right|} 0.09, \sqrt{\left|b_{d}\right|} 20 \mathrm{TeV}$ for generic $f$ s. In the case $\xi \sim \mathbf{3}, \mathbf{8} \in \mathrm{SU}(3)_{q}$ all corrections are proportional to the light mass, and $m_{*}$ above the $\mathrm{TeV}$ would suffice.

The CP-odd 4-fermion interactions contributing to $d_{n}$ are of the form $\bar{q} u \bar{q} d$ and do not constrain these scenarios further.

\subsection{Collider searches}

Additional constraints on these scenarios come from collider searches of the colored messengers $\Psi$, which generically correspond to one or more families of $\mathrm{SU}(2)_{w}$-singlet vector-like top or bottom quarks. At the LHC they are dominantly pair-produced by QCD interactions (single-production via the Yukawas is not competitive at present) and are expected to decay mainly into third generation quarks with $\mathrm{BR}(\Psi \rightarrow h t) \approx \mathrm{BR}\left(\Psi \rightarrow Z^{0} t\right) \approx \frac{1}{2} \mathrm{BR}(\Psi \rightarrow$ $\left.W^{+} b\right) \approx 25 \%$ for $\xi \sim \mathbf{3} \in \mathrm{SU}(3)_{u}$, whereas $\mathrm{BR}(\Psi \rightarrow h b) \approx \mathrm{BR}\left(\Psi \rightarrow Z^{0} b\right) \approx \frac{1}{2} \mathrm{BR}(\Psi \rightarrow$ $\left.W^{-} t\right) \approx 25 \%$ for $\xi \sim \mathbf{3} \in \mathrm{SU}(3)_{d}$. Current searches for heavy partners of the top and bottom quarks can be used to set an approximate lower bound $m_{*} \gtrsim 800-900 \mathrm{GeV}$ [60-63] on one-generation models.

These bounds should straightforwardly apply to the minimal 4D models of appendix A (with $\xi \sim \mathbf{3}$ of either $\mathrm{SU}(3)_{u}$ or $\left.\mathrm{SU}(3)_{d}\right)$. However, the warped 5D models introduced in the previous section have a more model-dependent collider phenomenology, and may or may not have light $\Psi$ s, as argued in section 3.3.

\footnotetext{
${ }^{8}$ Recall that, assuming $\bar{\theta}=0$, the contribution from pure SM physics is expected to be of order $\left|d_{n}\right| / e \sim$ $10^{-32} \mathrm{~cm}$ (see e.g. [59] and references therein).
} 


\section{Discussion}

What makes the strong CP problem stand out from the list of puzzles in particle physics is the absence of an obvious "environmental justification". While a small cosmological constant [64], the Planck-TeV hierarchy [65], the existence of dark matter and the rarity of anti-matter [66] may be argued to be essential to the existence of our universe, the smallness of the neutron EDM cries out for a dynamical explanation.

In this paper we discussed solutions to the strong CP problem based on spontaneous $\mathrm{CP}$ violation. The objective of these scenarios is to generate, after symmetry breaking, a CKM phase of order unity while simultaneously forbid large corrections to $\bar{\theta}$. This poses severe constraints on the way $\mathrm{CP}$ violation is communicated to quarks, and on flavorviolation beyond the Standard Model. These features are in sharp contrast to the QCD axion solution and should be viewed, in our opinion, as an important clue on the nature of the short distance physics.

Our key observation is that $\bar{\theta}$ is a flavor-singlet CP-odd parameter, whereas all known sources of $\mathrm{CP}$ violation - encoded in the CKM matrix - arise in flavor-violating processes. A robust solution to the strong $\mathrm{CP}$ problem must therefore ensure that the CP-odd scalars interact with the SM in such a way that all flavor-singlet CP-odd combinations of their vevs are suppressed, while the flavor-violating combinations are allowed to be large.

In a Supersymmetric world, finding theories of this type would be quite easy, because the SUSY non-renormalization theorems forbid perturbative contributions to $\bar{\theta}[26,27,67]$ (up to threshold effects). We can understand this result, and extend it to take into account non-perturbative contributions, using the spurion formalism of section 2.2 and holomorphicity of the Wilsonian action [68]: in a SUSY framework $\bar{\theta}$ is renormalized only if there exists a holomorphic, CP-odd, flavor-singlet combination of the spurions. ${ }^{9}$ Unfortunately, SUSY is broken, and one should find a way to protect $\bar{\theta}$ without it.

From a general non-Supersymmetric perspective, the most favorable scenarios are those (see figure 1) in which the dominant flavor-violating parameters in the visible sector are (in a suitable field basis) the CP-even proto-Yukawa matrices $y_{u, d}$, accounting for the quark mass hierarchy, and the CP-odd spurion(s) $\xi$. The quark Yukawas and the topological angle in the effective field theory are functions of these parameters, with $\xi \sim 1$ necessary to ensure a sizable CKM phase. We have seen that departures from the setup of figure 1 are strongly constrained, see section 2.2.1.

Our main result is a robust, model-independent criteria to ensure $|\bar{\theta}| \ll 1$, see section 2 . Simply stated, one just needs to build a scenario of the type represented in figure 1 and make sure that there exists either a unique $\xi$ charged under the fundamental or the adjoint of $\mathrm{SU}(3)_{u, d}$, or two CP-odd spurions $\xi_{u, d}$, one in $\mathrm{SU}(3)_{u}$ and one in $\mathrm{SU}(3)_{d}$. This is equivalent to state that $\mathrm{CPV}$ must be communicated to the SM via couplings involving colored messenger fields, the SM-neutral CP-odd scalars, and either $u$ and/or $d$; couplings to the quark doublet $q$ must be suppressed. If this simple requirement is met, the accidental symmetries of the low energy theory guarantee that $\bar{\theta}$ first arises at sixth order in the SM

\footnotetext{
${ }^{9}$ Thus, a flavor-singlet spurion, a sextet, a bi-fundamental, etc. would renormalize $\bar{\theta}$, whereas the choices $\xi \sim \mathbf{3}, \mathbf{8}$, etc. do not.
} 
Yukawas, comfortably below the bound (1.1), see table 1. Solutions that do not meet these criteria usually require fine-tuning or non-trivial anti-correlations between the (a priori independent) flavor-violating parameters.

It is interesting to note that our conditions are neither stronger nor weaker than Barr's conditions [20] for a vanishing tree-level $\bar{\theta}$; they are just different. There exist models in Barr's class that do not meet our criteria (for instance the one originally proposed by Nelson $[18,19]$, that has two CP-odd spurions, one of which resides in $\left.\mathrm{SU}(3)_{q}\right)$, and there are scenarios that satisfy our requirements but do not belong to Barr's class (such as the model of section 3 and some of those reviewed in appendix B). Yet, the simplest 4D scenarios that satisfy our criteria are of the type presented in [71], which also fall in Barr's class (see appendix A).

Current bounds on these models are dominated by flavor and electroweak data as well as LHC searches for top and bottom partners. These models provide an excellent example of well motivated new physics scenarios with potentially spectacular signatures at hadron colliders, but no obvious connection to the hierarchy problem. As a concrete example, consider a Supersymmetric realization of the 4D models of appendix A with flavoranarchic soft masses in the range $\widetilde{m} \sim 10^{3}-10^{5} \mathrm{TeV}$ and suppressed $A$-terms, as suggested in $[69,70]$. An analysis similar to that leading to (2.6) shows that with $\xi \sim \mathbf{3} \in \mathrm{SU}(3)_{d}$ - an hypothesis compatible with $\mathrm{SU}(5)$ unification $-\bar{\theta}$ dominantly arises from 2-loop diagrams involving gluinos. The latter are under control for $m_{*} / \widetilde{m} \lesssim 10^{-5}-10^{-4}$, that translates into $m_{*} \lesssim 10 \mathrm{TeV}$, a value within the reach of the LHC.

Following the criteria of section 2, a variety of novel solutions to the strong CP problem can be constructed in an extra dimensional setup (section 3 ). Here $\theta_{\mathrm{CKM}} \sim 1$ is obtained dynamically, as opposed to weakly-coupled $4 \mathrm{D}$ scenarios where this condition usually requires a special relation between two a priori independent parameters. These 5D models of "quark compositeness" find a natural implementation within composite Higgs models, that are independently motivated by the hierarchy problem. We discussed in detail a realistic scenario with "composite right-handed quarks", where CP violation is shined through the bulk by the scalar responsible for stabilizing the extra dimension. The model addresses both the naturalness of the weak scale and the strong CP problem, and is compatible with a new physics scale above a few $\mathrm{TeV}$ (see figure 3), with the electroweak precision constraints basically insensitive to the new ingredients introduced to solve the strong CP problem. Scenarios with "composite doublets" can be realized along similar lines.

We showed in section 3.4 that all our models (the 4D and 5D models of appendix A and section 3) belong to the same, larger class of CFTs reproducing the picture of figure 1 , but we have not been able to prove that the opposite is also true. It would be interesting to find realistic models consistent with our requirements which are not of the type described by eq. (3.14).

In the models depicted in figure 1, flavor-violation beyond the SM is controlled by $\xi$, and this points to interesting model-dependent correlations between the neutron EDM and flavor-violating observables, that can potentially be tested experimentally. For example, 5D models with $\xi \in \mathrm{SU}(3)_{d}$ and $m_{*}$ in the multi-TeV range induce large flavor-violating effects in the down sector and a potentially testable neutron EDM from dimension-6 operators. 
On the other hand, if $\xi$ is charged under $\mathrm{SU}(3)_{u}$ and $m_{*}$ sufficiently large, a $\bar{\theta}$ within the reach of future experiments may well be the only signature.

$\mathrm{CP}$ violation may leak in the lepton sector via a spurion carrying lepton flavor indices in a way analogous to the one described here for quarks. Our results show that, as long as exotic lepto-quark interactions are suppressed, CPV in the lepton sector is under control provided the electroweak topological angle is $\left|\bar{\theta}_{w}\right| \lesssim 10^{-5}$ - a much weaker condition that $|\bar{\theta}| \lesssim 10^{-10}$. Leptogenesis thus appears as a natural mechanism to generate a matter anti-matter asymmetry in these models.

\section{Acknowledgments}

We would like to thank R. Sundrum and R. Mohapatra for useful discussions, and G. Senjanovic for comments on the first version of this work. The paper was originally submitted to the ArXiv in December of 2014 (arXiv:1412.3805) and, for reasons that transcend human intellect, only sent to JHEP on November 28, 2016. In the published version we updated our analysis in section 4. This work was supported in part by NSF Grant No. PHY-1315155 and by the Maryland Center for Fundamental Physics.

\section{A 4D models}

The simplest models realizing the setup of figure 1 can be written in the notation of eq. (3.14) as

$$
O_{u}=\bar{q} \widetilde{H} u, \quad O_{d}=\bar{q} H d, \quad O=\bar{Q} \Psi \quad(Q=u \text { and } / \text { or } d)
$$

where $\Psi$ is a vector-like fermion with mass $m_{\Psi}$ and appropriate charges under the SM, and the CP-odd spurion is automatically in the fundamental of $\mathrm{SU}(3)_{u, d}$. The case $Q=d$ and $\Sigma$ a fundamental scalar (i.e. $d_{\Sigma}=1$ ) has been first presented in [71], see [26, 27] for a realistic SUSY version. The models (A.1) satisfy Barr's criteria [20], which appear here as a consequence of our requirements.

The scenarios (A.1), including SUSY extensions, are appealing because very minimal. We emphasize however that

$$
\left|Y_{Q} \lambda\langle\Sigma\rangle\right| \lesssim m_{\Psi} \lesssim|\lambda\langle\Sigma\rangle|
$$

must be arranged to guarantee perturbativity. To see this note that a large mixing $\Psi-Q$ is needed to get $\theta_{\mathrm{CKM}} \sim 1$, and this requires $m_{\Psi} \lesssim \lambda\langle\Sigma\rangle$. On the other hand, taking $m_{\Psi} / \lambda\langle\Sigma\rangle \ll 1$ effectively enhances the coupling between the physical heavy modes of mass $m_{*}^{2}=m_{\Psi}^{2}+|\lambda \Sigma|^{2}$ (a combination of $Q, \Psi$ ) and $\bar{q} H$ or $\bar{q} \widetilde{H}$. The latter coupling is $Y_{Q} U \lambda\langle\Sigma\rangle / m_{\Psi}$, with $U$ a unitary matrix. Barring accidental cancellations among $y_{u, d}$ and $\lambda\langle\Sigma\rangle$, requiring perturbativity below the cutoff thus sets an upper bound on $Y_{Q} \lambda\langle\Sigma\rangle / m_{\Psi}$ of order unity, that reads as in (A.2).

In models with $m_{\Psi}$ a bare mass defined at the UV cutoff $\Lambda$, the condition (A.2) is the result of a peculiar choice of UV parameters. In fact, because the couplings $\lambda$ and 
$m_{\Psi}$ run very differently from the cutoff down to $\langle\Sigma\rangle \ll \Lambda$, from an effective field theory perspective (A.2) appears as a remarkable coincidence. This is even more so if $\Sigma$ is a fermion bilinear. On the other hand, if for example

$$
m_{\Psi}=\lambda^{\prime}\langle S\rangle,
$$

for some scalar $S$ getting a vev comparable to that of $\Sigma$, then (A.2) becomes $\lambda(\Lambda) \sim \lambda^{\prime}(\Lambda)$, which is a perfectly legitimate and radiatively stable assumption on the short distance dynamics. Unfortunately, in these latter models the minimality of (A.1) is partially lost.

In our warped model the condition $m_{\Psi} \sim|\lambda\langle\Sigma\rangle|$ is dynamically generated, see (3.15), and hence (A.2) is automatic.

\section{B On the previous literature}

HS. In SUSY models, the dominant contributions to the neutron EDM come below the SUSY-breaking scale, and the authors of refs. [26, 27] showed that these can be made small provided (1) CP violation occurs at a scale $m_{\mathrm{CP}}$ much larger than SUSY breaking, (2) SUSY-breaking is communicated via some flavor-invariant mediator (e.g. gauge mediation), and (3) the SUSY-breaking sector interacts weakly with the CP-violating dynamics. Under these reasonable assumptions, the IR physics respects MFV, and contributions to $\bar{\theta}$ are utterly small $[2,26,27,72,73]$. This regime coincides with the limit of exact MFV, and trivially meets our criteria.

Refs. $[26,27]$ generates the CKM phase either with the Nelson-Barr mechanism, which we discussed in appendix A, or by invoking a non-perturbative interaction between the SM quarks and messenger fields that directly couple to the CP-odd scalar. For the latter mechanism to work in the specific model discussed in [26, 27], the quark-messenger coupling must become strong exactly at the messenger mass scale, which is an independent relevant parameter. Besides bringing a calculability issue on the table, this poses a coincidence problem (mentioned in appendix A) that was not addressed in [26, 27]. The 5D models presented here employ a similar mechanism, but solve these issues by constructing calculable dual scenarios with composite quarks.

HPSS. In the $5 \mathrm{D}$ model $[28]^{10} \mathrm{NDA}$ suggests that both scales $\lambda \Sigma, m_{*}$ are naturally set by the $5 \mathrm{D}$ cutoff, so the second condition in (1.1) is obtained naturally. This is analogous to what happens in the $5 \mathrm{D}$ example of section 3.

Using a (dual) 4D language, we may view $[28,29]$ as models in which $\mathrm{CP}$ is spontaneously broken at $O(1)$ by a strong dynamics (i.e. the bulk) that violates flavor. Our analysis suggests that in such a situation there is a priori no small parameter that suppresses $\bar{\theta}$. Indeed, in order to reduce unwanted UV contributions to $\bar{\theta}$, additional assumptions have been made in [28] (and [29]). The authors discuss 3 possibilities. First, the vev of the bulk CP-odd scalar may be much smaller than the cutoff - this requires a fine-tuning. Second, the bulk CP-odd scalar may be assumed to have no overlap with the branes (by the automatic P-invariance of the bulk, $\bar{\theta}$ can only appear on the boundaries), though no

\footnotetext{
${ }^{10} \mathrm{~A}$ realization on a warped background was presented in [29].
} 


\begin{tabular}{|c|cc|}
\hline & $\mathrm{SU}(n)$ & $\mathrm{U}(1)_{\xi}$ \\
\hline$Q_{i L}$ & $\mathbf{n}$ & $\pm \frac{1}{2}$ \\
$Q_{j R}$ & $\mathbf{n}$ & $\mathbf{0}$ \\
\hline
\end{tabular}

Table 2. Field content of a CP-violating sector, with flavor indices $i, j=1,2$.

simple way to realize this possibility exists. Third, the CP-odd scalar may be promoted to a $8 \in \mathrm{SU}(3)_{Q}$, which is one of the possibilities discussed in table 1 . In this latter case $\bar{\theta}$ can be naturally below the current bounds, but the solution of the flavor puzzle proposed in $[28,29]$ should be re-considered.

\section{A minimal confining CP-violating sector}

Here we present a minimal CPV sector. This can be employed, together with the class of models captured by (3.14), to build explicit realizations of figure 1 .

An elegant way to achieve CPV at a scale $m_{\mathrm{CP}}$ parametrically smaller than the UV cutoff $\Lambda$ is to introduce chiral fermions $Q$ charged under a confining strong dynamics and a weakly gauged $\mathrm{U}(1)_{\xi}$. A minimal field content is shown in table 2 . This is basically a copy of 2 -flavor $\mathrm{QCD}$, with $\mathrm{U}(1)_{\xi}$ generated by $\sigma^{3}$ of $\mathrm{SU}(2)_{L}$, and

$$
\Sigma_{i j}=\overline{Q_{j R}} Q_{i L}
$$

The gauged $\mathrm{SU}(n)$ gets strong at a scale $m_{\mathrm{CP}} \ll \Lambda$ and produces 3 Goldstone modes, one combination of which is eaten by the $\mathrm{U}(1)_{\xi}$ vector. We can perform gauge rotations such that the physical Nambu-Goldstone modes are the two angles $\pi_{1,2}$ defined by

$$
\Sigma \propto\left(\begin{array}{cc}
e^{i \pi_{2}} \cos \pi_{1} & e^{-i \pi_{2}} \sin \pi_{1} \\
-e^{i \pi_{2}} \sin \pi_{1} & e^{-i \pi_{2}} \cos \pi_{1}
\end{array}\right) .
$$

In the basis in which the Lagrangian has all real coefficients, $\mathrm{CP}$ acts as $\Sigma \rightarrow L \Sigma$, with $L \in \mathrm{U}(1)_{\xi}$ - up to real chiral rotations. A careful analysis shows that $\mathrm{CP}$ is broken if and only if $2 \pi_{2} \neq \pi n(n=0, \pm 1, \pm 2, \cdots)$, i.e. if $\sin 2 \pi_{2} \neq 0$.

The global $\mathrm{SU}(2)_{L} \times \mathrm{SU}(2)_{R}$ is explicitly broken by the gauged $\mathrm{U}(1)_{\xi}$, the irrelevant coupling $\lambda \Sigma O$ of (3.14), and cutoff-suppressed operators such as $Q Q Q Q$. Yet, the weak gauging does not induce a potential for $\pi_{1,2}$ because $\mathrm{U}(1)_{\xi}$ does not simultaneously break $\mathrm{SU}(2)_{L}$ and $\mathrm{SU}(2)_{R}$. To see this, observe the effect of $\mathrm{U}(1)_{\xi}$ may be parametrized by a spurion $\sigma_{3}$ in the adjoint of $\mathrm{SU}(2)_{L}$, and that there is no potential we can build with $\Sigma, \sigma_{3}$ that is formally invariant under the chiral symmetry. We conclude that only $\lambda \Sigma O$ and $Q Q Q Q$ are relevant to vacuum alignment. The effect of $\lambda \Sigma O$ is at least one-loop, so we conservatively assume the potential is dominated by the 4 -fermion operators. For generic, real coefficients of $Q Q Q Q$ one finds that $\mathrm{CP}$ is spontaneously broken and $\pi_{1,2} \sim 1 .{ }^{11}$ This generates a complex tadpole $\lambda\langle\Sigma\rangle$, see (3.9).

\footnotetext{
${ }^{11}$ Analogously, QCD spontaneously breaks CP [74] for generic quark masses.
} 
It is easy to verify that all contributions to $\bar{\theta}$ from physics $\gtrsim m_{\mathrm{CP}}$ are small. In fact, these are naturally suppressed whenever CP violation is soft, i.e. $\langle\Sigma\rangle \sim m_{\mathrm{CP}}^{d_{\Sigma}}$ with $m_{\mathrm{CP}} \ll \Lambda \sim 10^{18} \mathrm{GeV}$. In particular, effects controlled by $\Sigma^{\dagger} \Sigma$ (such as a bare $\bar{\theta}$ ) are negligible as long as

$$
\left(\frac{m_{\mathrm{CP}}}{\Lambda}\right)^{d_{\Sigma}}<10^{-5}
$$

with $d_{\Sigma}$ the scaling dimension of $\Sigma$. For this particular CPV sector $d_{\Sigma} \simeq 3$, and it is sufficient to require $m_{\mathrm{CP}} \lesssim 10^{-2} \Lambda$.

Below the scale $m_{\mathrm{CP}}$ the CP-violating sector is described by the Goldstone modes. Loops of $\pi_{1,2}$ could in principle give a dangerous correction to $\bar{\theta}$, but these can be made parametrically small by taking $d_{\Sigma}+d_{O}>4$. Under these conditions, CP violation at scales $<m_{\mathrm{CP}}$ is controlled by the complex matrix $\xi \propto J$, realizing the framework of figure 1 .

A final comment is in order. The CP-violating dynamics has hadrons at the scale $m_{\mathrm{CP}}$, as well as Goldstone modes of mass $m_{\pi_{1,2}} \sim m_{\mathrm{CP}}^{2} / \Lambda \ll m_{\mathrm{CP}}$ that are very weakly coupled to ordinary matter and were never brought to thermal equilibrium. The Goldstone modes are unstable and do not represent a cosmological hazard. The abundance of the $\mathrm{SU}(n)$ baryons is not calculable. Yet, if the re-heating temperature of the universe is postulated to be below $m_{\mathrm{CP}}$ in order to avoid any complication with domain walls generated at $\mathrm{CP}$ breaking, then one finds that the present-day population of baryons is negligible.

Open Access. This article is distributed under the terms of the Creative Commons Attribution License (CC-BY 4.0), which permits any use, distribution and reproduction in any medium, provided the original author(s) and source are credited.

\section{References}

[1] Particle Data Group collaboration, K.A. Olive et al., Review of Particle Physics, Chin. Phys. C 38 (2014) 090001 [inSPIRE].

[2] J.R. Ellis and M.K. Gaillard, Strong and Weak CP-violation, Nucl. Phys. B 150 (1979) 141 [INSPIRE].

[3] I.B. Khriplovich, Quark Electric Dipole Moment and Induced $\theta$ Term in the Kobayashi-Maskawa Model, Phys. Lett. B 173 (1986) 193 [InSPIRE].

[4] A. Czarnecki and B. Krause, Neutron electric dipole moment in the standard model: Valence quark contributions, Phys. Rev. Lett. 78 (1997) 4339 [hep-ph/9704355] [INSPIRE].

[5] E. Vicari and H. Panagopoulos, Theta dependence of $\mathrm{SU}(N)$ gauge theories in the presence of a topological term, Phys. Rept. 470 (2009) 93 [arXiv:0803.1593] [inSPIRE].

[6] J. Engel, M.J. Ramsey-Musolf and U. van Kolck, Electric Dipole Moments of Nucleons, Nuclei and Atoms: The Standard Model and Beyond, Prog. Part. Nucl. Phys. 71 (2013) 21 [arXiv: 1303.2371] [INSPIRE].

[7] R.D. Peccei and H.R. Quinn, CP Conservation in the Presence of Instantons, Phys. Rev. Lett. 38 (1977) 1440 [INSPIRE].

[8] R.D. Peccei and H.R. Quinn, Constraints Imposed by CP Conservation in the Presence of Instantons, Phys. Rev. D 16 (1977) 1791 [INSPIRE]. 
[9] S. Weinberg, A New Light Boson?, Phys. Rev. Lett. 40 (1978) 223 [InSPIRE].

[10] F. Wilczek, Problem of Strong $p$ and $t$ Invariance in the Presence of Instantons, Phys. Rev. Lett. 40 (1978) 279 [INSPIRE].

[11] H. Georgi and I.N. McArthur, Instantons And The Mu Quark Mass, HUTP-81/A011 (1981).

[12] K. Choi, C.W. Kim and W.K. Sze, Mass Renormalization by Instantons and the Strong CP Problem, Phys. Rev. Lett. 61 (1988) 794 [INSPIRE].

[13] T. Banks, Y. Nir and N. Seiberg, Missing (up) mass, accidental anomalous symmetries and the strong CP problem, in Proceedings of second IFT Workshop: Yukawa couplings and the origin of mass, Gainesville U.S.A. (1994), pg. 26 [hep-ph/9403203] [INSPIRE].

[14] S. Aoki et al., Review of lattice results concerning low-energy particle physics, Eur. Phys. J. C 74 (2014) 2890 [arXiv:1310.8555] [INSPIRE].

[15] H. Georgi, A Model of Soft CP Violation, Hadronic J. 1 (1978) 155 [INSPIRE].

[16] M.A.B. Beg and H.S. Tsao, Strong P, T Noninvariances in a Superweak Theory, Phys. Rev. Lett. 41 (1978) 278 [INSPIRE].

[17] R.N. Mohapatra and G. Senjanović, Natural Suppression of Strong $p$ and $t$ Noninvariance, Phys. Lett. B 79 (1978) 283 [INSPIRE].

[18] A.E. Nelson, Naturally Weak CP-violation, Phys. Lett. B 136 (1984) 387 [INSPIRE].

[19] A.E. Nelson, Calculation of $\theta$ Barr, Phys. Lett. B 143 (1984) 165 [InSPIRE].

[20] S.M. Barr, Solving the Strong CP Problem Without the Peccei-Quinn Symmetry, Phys. Rev. Lett. 53 (1984) 329 [INSPIRE].

[21] K.S. Babu and R.N. Mohapatra, A Solution to the Strong CP Problem Without an Axion, Phys. Rev. D 41 (1990) 1286 [inSPIRE].

[22] S.M. Barr, D. Chang and G. Senjanović, Strong CP problem and parity, Phys. Rev. Lett. 67 (1991) 2765 [INSPIRE].

[23] R. Kuchimanchi, Solution to the strong CP problem: Supersymmetry with parity, Phys. Rev. Lett. 76 (1996) 3486 [hep-ph/9511376] [INSPIRE].

[24] R.N. Mohapatra and A. Rasin, Simple supersymmetric solution to the strong CP problem, Phys. Rev. Lett. 76 (1996) 3490 [hep-ph/9511391] [INSPIRE].

[25] R.N. Mohapatra, A. Rasin and G. Senjanović, $P, C$ and strong $C P$ in left-right supersymmetric models, Phys. Rev. Lett. 79 (1997) 4744 [hep-ph/9707281] [INSPIRE].

[26] G. Hiller and M. Schmaltz, Solving the strong CP problem with supersymmetry, Phys. Lett. B 514 (2001) 263 [hep-ph/0105254] [INSPIRE].

[27] G. Hiller and M. Schmaltz, Strong weak CP hierarchy from nonrenormalization theorems, Phys. Rev. D 65 (2002) 096009 [hep-ph/0201251] [INSPIRE].

[28] R. Harnik, G. Perez, M.D. Schwartz and Y. Shirman, Strong CP, flavor and twisted split fermions, JHEP 03 (2005) 068 [hep-ph/0411132] [INSPIRE].

[29] C. Cheung, A.L. Fitzpatrick and L. Randall, Sequestering CP-violation and GIM-Violation with Warped Extra Dimensions, JHEP 01 (2008) 069 [arXiv:0711.4421] [INSPIRE].

[30] R. Kuchimanchi, P/CP Conserving CP/P Violation Solves Strong CP Problem, Phys. Rev. D 82 (2010) 116008 [arXiv: 1009.5961] [INSPIRE]. 
[31] S. Antusch, M. Holthausen, M.A. Schmidt and M. Spinrath, Solving the Strong CP Problem with Discrete Symmetries and the Right Unitarity Triangle, Nucl. Phys. B 877 (2013) 752 [arXiv: 1307.0710] [INSPIRE].

[32] H.-Y. Cheng, The Strong CP Problem Revisited, Phys. Rept. 158 (1988) 1 [INSPIRE].

[33] Z. Berezhiani, L. Gianfagna and M. Giannotti, Strong CP problem and mirror world: The Weinberg-Wilczek axion revisited, Phys. Lett. B 500 (2001) 286 [hep-ph/0009290] [INSPIRE].

[34] A. Hook, Anomalous solutions to the strong CP problem, Phys. Rev. Lett. 114 (2015) 141801 [arXiv:1411.3325] [INSPIRE].

[35] M. Kamionkowski and J. March-Russell, Planck scale physics and the Peccei-Quinn mechanism, Phys. Lett. B 282 (1992) 137 [hep-th/9202003] [INSPIRE].

[36] S.M. Barr and D. Seckel, Planck scale corrections to axion models, Phys. Rev. D 46 (1992) 539 [INSPIRE].

[37] R. Holman, S.D.H. Hsu, T.W. Kephart, E.W. Kolb, R. Watkins and L.M. Widrow, Solutions to the strong CP problem in a world with gravity, Phys. Lett. B 282 (1992) 132 [hep-ph/9203206] [INSPIRE].

[38] K.-w. Choi, D.B. Kaplan and A.E. Nelson, Is CP a gauge symmetry?, Nucl. Phys. B 391 (1993) 515 [hep-ph/9205202] [INSPIRE].

[39] M. Dine, R.G. Leigh and D.A. MacIntire, Of CP and other gauge symmetries in string theory, Phys. Rev. Lett. 69 (1992) 2030 [hep-th/9205011] [INSPIRE].

[40] A. Rasin, Diagonalization of quark mass matrices and the Cabibbo-Kobayashi-Maskawa matrix, hep-ph/9708216 [INSPIRE].

[41] H. Georgi, T. Tomaras and A. Pais, Strong CP-violation without instantons, Phys. Rev. D 23 (1981) 469 [INSPIRE].

[42] A. Dedes and M. Pospelov, Theta angle versus CP-violation in the leptonic sector, Phys. Rev. D 61 (2000) 116010 [hep-ph/9912293] [INSPIRE].

[43] R. Kuchimanchi, Leptonic CP problem in left-right symmetric model, Phys. Rev. D 91 (2015) 071901 [arXiv: 1408.6382] [INSPIRE].

[44] L. Randall and R. Sundrum, A Large mass hierarchy from a small extra dimension, Phys. Rev. Lett. 83 (1999) 3370 [hep-ph/9905221] [INSPIRE].

[45] W.D. Goldberger and M.B. Wise, Modulus stabilization with bulk fields, Phys. Rev. Lett. 83 (1999) 4922 [hep-ph/9907447] [INSPIRE].

[46] M. Redi and A. Weiler, Flavor and CP Invariant Composite Higgs Models, JHEP 11 (2011) 108 [arXiv: 1106.6357] [INSPIRE].

[47] R. Rattazzi and A. Zaffaroni, Comments on the holographic picture of the Randall-Sundrum model, JHEP 04 (2001) 021 [hep-th/0012248] [INSPIRE].

[48] G. Cacciapaglia, C. Csáki, J. Galloway, G. Marandella, J. Terning and A. Weiler, A GIM Mechanism from Extra Dimensions, JHEP 04 (2008) 006 [arXiv:0709.1714] [INSPIRE].

[49] L. Vecchi, Phenomenology of a light scalar: the dilaton, Phys. Rev. D 82 (2010) 076009 [arXiv: 1002.1721] [INSPIRE]. 
[50] J.A. Aguilar-Saavedra, R. Benbrik, S. Heinemeyer and M. Pérez-Victoria, Handbook of vectorlike quarks: Mixing and single production, Phys. Rev. D 88 (2013) 094010 [arXiv: 1306.0572] [INSPIRE].

[51] C.-Y. Chen, S. Dawson and E. Furlan, Vector-like Fermions and Higgs Effective Field Theory Revisited, arXiv:1703.06134 [INSPIRE].

[52] M. Baak et al., The Electroweak Fit of the Standard Model after the Discovery of a New Boson at the LHC, Eur. Phys. J. C 72 (2012) 2205 [arXiv:1209.2716] [InSPIRE].

[53] J. de Blas et al., Electroweak precision observables and Higgs-boson signal strengths in the Standard Model and beyond: present and future, JHEP 12 (2016) 135 [arXiv:1608.01509] [INSPIRE].

[54] UTfit collaboration, M. Bona et al., Model-independent constraints on $\Delta F=2$ operators and the scale of new physics, JHEP 03 (2008) 049 [arXiv:0707.0636] [INSPIRE].

[55] G. Isidori, Y. Nir and G. Perez, Flavor Physics Constraints for Physics Beyond the Standard Model, Ann. Rev. Nucl. Part. Sci. 60 (2010) 355 [arXiv:1002.0900] [inSPIRE].

[56] J. Charles et al., Current status of the Standard Model CKM fit and constraints on $\Delta F=2$ New Physics, Phys. Rev. D 91 (2015) 073007 [arXiv:1501.05013] [InSPIRE].

[57] B. Grinstein, R.P. Springer and M.B. Wise, Strong Interaction Effects in Weak Radiative $\bar{B}$ Meson Decay, Nucl. Phys. B 339 (1990) 269 [INSPIRE].

[58] G. D'Ambrosio, G.F. Giudice, G. Isidori and A. Strumia, Minimal flavor violation: An Effective field theory approach, Nucl. Phys. B 645 (2002) 155 [hep-ph/0207036] [INSPIRE].

[59] C.-Y. Seng, Reexamination of The Standard Model Nucleon Electric Dipole Moment, Phys. Rev. C 91 (2015) 025502 [arXiv: 1411.1476] [INSPIRE].

[60] CMS collaboration, Search for vector-like charge $2 / 3$ T quarks in proton-proton collisions at $\sqrt{s}=8 \mathrm{TeV}$, Phys. Rev. D 93 (2016) 012003 [arXiv:1509.04177] [inSPIRE].

[61] CMS collaboration, Search for pair-produced vectorlike B quarks in proton-proton collisions at $\sqrt{s}=8 \mathrm{TeV}$, Phys. Rev. D 93 (2016) 112009 [arXiv:1507.07129] [INSPIRE].

[62] ATLAS collaboration, Search for production of vector-like quark pairs and of four top quarks in the lepton-plus-jets final state in pp collisions at $\sqrt{s}=8 \mathrm{TeV}$ with the ATLAS detector, JHEP 08 (2015) 105 [arXiv: 1505.04306] [INSPIRE].

[63] ATLAS collaboration, Search for pair production of heavy vector-like quarks decaying to high- $p_{T} W$ bosons and $b$ quarks in the lepton-plus-jets final state in pp collisions at $\sqrt{s}=13$ TeV with the ATLAS detector, ATLAS-CONF-2016-102 (2016).

[64] S. Weinberg, Anthropic Bound on the Cosmological Constant, Phys. Rev. Lett. 59 (1987) 2607 [INSPIRE].

[65] V. Agrawal, S.M. Barr, J.F. Donoghue and D. Seckel, The Anthropic principle and the mass scale of the standard model, Phys. Rev. D 57 (1998) 5480 [hep-ph/9707380] [INSPIRE].

[66] M. Tegmark, A. Aguirre, M. Rees and F. Wilczek, Dimensionless constants, cosmology and other dark matters, Phys. Rev. D 73 (2006) 023505 [astro-ph/0511774] [INSPIRE].

[67] J.R. Ellis, S. Ferrara and D.V. Nanopoulos, CP Violation and Supersymmetry, Phys. Lett. B 114 (1982) 231 [INSPIRE].

[68] N. Seiberg, Naturalness versus supersymmetric nonrenormalization theorems, Phys. Lett. B 318 (1993) 469 [hep-ph/9309335] [INSPIRE]. 
[69] A. Arvanitaki, N. Craig, S. Dimopoulos and G. Villadoro, Mini-Split, JHEP 02 (2013) 126 [arXiv: 1210.0555] [INSPIRE].

[70] N. Arkani-Hamed, A. Gupta, D.E. Kaplan, N. Weiner and T. Zorawski, Simply Unnatural Supersymmetry, arXiv:1212.6971 [INSPIRE].

[71] L. Bento, G.C. Branco and P.A. Parada, A Minimal model with natural suppression of strong CP-violation, Phys. Lett. B 267 (1991) 95 [INSPIRE].

[72] M. Dugan, B. Grinstein and L.J. Hall, CP Violation in the Minimal $N=1$ Supergravity Theory, Nucl. Phys. B 255 (1985) 413 [InSPIRE].

[73] I.B. Khriplovich and A.I. Vainshtein, Infinite renormalization of Theta term and Jarlskog invariant for CP-violation, Nucl. Phys. B 414 (1994) 27 [hep-ph/9308334] [InSPIRE].

[74] R.F. Dashen, Some features of chiral symmetry breaking, Phys. Rev. D 3 (1971) 1879 [INSPIRE]. 